RICARDO CÉSAR CORRÊA

\title{
WDSC: Wi-Fi Direct with Spontaneous Connection
}

São Paulo

2016 


\section{RICARDO CÉSAR CORRÊA}

\section{WDSC: Wi-Fi Direct with Spontaneous Connection}

Dissertação apresentada à Escola de Artes, Ciências e Humanidades da Universidade de São Paulo para obtenção do título de Mestre em Ciências pelo Programa de Pós-graduação em Sistemas de Informação.

Área de Concentração: Desenvolvimento de Sistemas

Versão corrigida contendo as alterações solicitadas pela comissão julgadora em 30 de junho de 2016. A versão original encontra-se em acervo reservado na Biblioteca da EACHUSP e na Biblioteca Digital de Teses e Dissertações da USP (BDTD), de acordo com a Resolução CoPGr 6018, de 13 de outubro de 2011.

Orientador: Prof. Dr. Luciano Vieira de Araújo

São Paulo

2016 
Autorizo a reprodução e divulgação total ou parcial deste trabalho, por qualquer meio convencional ou eletrônico, para fins de estudo e pesquisa, desde que citada a fonte.

CATALOGAÇÃO-NA-PUBLICAÇÃO

(Universidade de São Paulo. Escola de Artes, Ciências e Humanidades. Biblioteca)

\section{Corrêa, Ricardo César}

WDSC : Wi-fi direct with spontaneous connection / Ricardo

César Corrêa ; orientador, Luciano Vieira de Araújo. - São Paulo, 2016

$57 \mathrm{f}$.

Dissertação (Mestrado em Ciências) - Programa de PósGraduação em Sistemas de Informação, Escola de Artes, Ciências e Humanidades, Universidade de São Paulo

Versão corrigida

1. Wireless. 2. Computação móvel. 3. Internet. 4. Wi-fi direct. I. Araújo, Luciano Vieira de, orient. II. Título

$$
\text { CDD 22.ed.- } 005.2
$$


Dissertação de autoria de Ricardo César Corrêa, sob o título "WDSC: Wi-Fi Direct with Spontaneous Connection", apresentada à Escola de Artes, Ciências e Humanidades da Universidade de São Paulo, para obtenção do título de Mestre em Ciências pelo Programa de Pós-graduação em Sistemas de Informação, na área de concentração Desenvolvimento de Sistemas, aprovada em 30 de Junho de 2016 pela comissão julgadora constituída pelos doutores:

\section{Prof. Dr. Luciano Vieira de Araújo}

Presidente

Instituição: Escola de Artes, Ciências e Humanidades - Universidade de São Paulo

Profa. Dra. Fátima de Lourdes dos Santos Nunes Marques

Instituição: Escola de Artes, Ciências e Humanidades - Universidade de São Paulo

\section{Prof. Dr. Ivan Torres Pisa}

Instituição: Departamento de Informática em Saúde - Universidade Federal de São Paulo

\section{Prof. Dr. Márcio Katsumi Oikawa}

Instituição: Centro de Matemática, Computação e Cognição - Universidade Federal do ABC 


\section{AGRADECIMENTOS}

Agradecimentos serão, nesse momento, meu singelo reconhecimento a aqueles que, de forma direta ou indireta, me ajudaram a desenvolver este trabalho que é, para mim, o resultado de uma pós-graduação intensa e gratificante.

Em primeiro lugar, agradeço ao meu orientador Dr. Luciano Vieira de Araújo que me acolheu sem receio e me possibilitou desenvolver um grande trabalho por meio de sua admirável e incansável dedicação em me orientar minuciosamente em todos os quesitos acadêmicos, o que exigiu paciência e, sobretudo, dedicação a sua atividade profissional. A Dra. Violeta Sun que, não só me orientou durante a graduação, como também me incentivou e encorajou a prosseguir em busca de novos desafios na área acadêmica.

Também aos professores do Programa de Pós-graduação de Sistemas de Informação, que exigiram dedicação aos estudos. Aos professores da banca de qualificação, Dr. Márcio Katsumi Oikawa e Dra. Fátima de Lourdes dos Santos Nunes Marques, pela gentileza da participação e pelos comentários necessários. Aos professores da banca de defesa Dra. Fátima Nunes Marques, Dr. Ivan Torres de Pisa e Dr. Márcio Katsumi Oikawa pela gentileza da participação e contribuição a esse trabalho.

A Dotz por me fornecer a flexibilidade necessária para que pudesse desenvolver este trabalho paralelamente as atividades profissionais.

Aos colegas e amigos de mestrado que me aconselharam e compartilharam alegrias e preocupações durante o curso, em especial, ao Marcos de Freitas Júnior. 
"O único lugar onde o sucesso vem antes do trabalho é no dicionário." Albert Einstein. 


\section{Resumo}

CÉSAR CORRÊA, Ricardo. WDSC: Wi-Fi Direct with Spontaneous Connection. 2016. 56 f. Dissertação (Mestrado em Ciências) - Escola de Artes, Ciências e Humanidades, Universidade de São Paulo, São Paulo, 2016.

O consumo de dados em dispositivos móveis cresce em taxas que dobram a cada ano. Tal crescimento demonstra como os dispositivos móveis ganham importância e como eles têm sido usados no dia a dia da população. Entretanto, o acesso à Internet ainda possui alto custo e nem sempre está disponível. Esse cenário apresenta o desafio de mantermos o uso dos dispositivos móveis acessível e viável, mesmo quando a Internet não esteja disponível. A comunicação direta entre os dispositivos é vista como alternativa para comunicação sem Internet e, consequentemente, para a redução do tráfego de dados nas redes de celulares. Além disso, ela pode ser útil para manter os dispositivos comunicando entre si mesmo desconectados da Internet. O protocolo Wi-Fi Direct (WD) é uma referência para esse tipo de comunicação, uma vez que seu protocolo não demanda infraestrutura de rede pré-estabelecida para que a comunicação aconteça e utiliza hardware padrão para comunicação Wi-Fi. Apesar dessas vantagens, seu uso é pouco difundido, o que o torna desconhecido pelos usuários, sendo uma das possíveis causas a complexidade de uso. De forma diferente, a conexão com a Internet, que acontece de forma quase espontânea, no WD as partes envolvidas precisam se envolver ativamente, na escolha e autorização, para que a conexão seja estabelecida. A complexidade da comunicação entre dispositivo pode ser constatada quando os usuários utilizam a Internet para enviar dados mesmo que o dispositivo alvo esteja ao lado da origem. Para superar esse obstáculo, a conexão entre os dispositivos deve ser tão ou mais simples do que a ação de enviar um e-mail ou uma mensagem instantânea usando a Internet. Para lidar com esse cenário, este trabalho propõe uma alternativa para comunicação entre dispositivos próximos que não demanda autorização manual prévia a cada conexão com um dispositivo, e que, ainda assim, permita que o usuário decida se quer ou não usar os dados transmitidos por uma conexão. Para isso definimos uma arquitetura que possibilite a conexão e transferência de dados entre os dispositivos próximos sem a necessidade de intervenção prévia do usuário e implementamos o conceito proposto no sistema operacional para dispositivos móveis Android.

Palavras-chave: Sistemas de comunicação móvel. Comunicação Wireless. Conexão espontânea. Wi-Fi Direct. 


\begin{abstract}
CÉSAR CORRÊA, Ricardo. WDSC: Wi-Fi Direct with Spontaneous Connection. 2016. 56 p. Dissertation (Master of Science) - School of Arts, Sciences and Humanities, University of São Paulo, São Paulo, 2016.

The consumption data on mobile devices is growing at rates double every year. This growth demonstrates how mobile devices gain importance and how they have been used in everyday life of the population. However, the internet also has high cost and is not always available. This scenario presents the challenge of maintaining the use of affordable and viable mobile devices even when the Internet is not available. Direct communication between the devices is regarded as no alternative for internet communication and, consequently, to reduce the data traffic in cellular networks. Furthermore, it can be useful to keep the devices communicating among themselves disconnected from the Internet. The Wi-Fi Direct (WD) protocol is a reference to this type of communication, since its protocol does not require pre-established network infrastructure for communication to happen and uses standard hardware for Wi-Fi communication. Despite these advantages, its use is not widespread, making it unknown to the users, one of the possible causes of the complexity of use. Differently, the connection to the Internet, which happens almost spontaneously, the WD parties need to engage actively in the selection and authorization for the connection to be established. The complexity of communication between device can be found when users use the Internet to send data even if the target device is adjacent to the source. To overcome this obstacle, the connection between the devices should be as or more simple than the action of sending an email or an instant message using the Internet. To handle this scenario, this paper proposes an alternative for communication between nearby devices that does not require prior manual authorization for each connection to a device, and that still allow the user to decide whether or not to use the data transmitted by a connection. For this we define an architecture that enables the connection and data transfer between nearby devices without the need for prior user intervention and implement the concept proposed in the Android operating system for mobile devices.
\end{abstract}

Keywords: Mobile communication systems. Wireless communication. Spontaneous Connection. Wi-Fi Direct. 


\section{Lista de figuras}

Figura 1 - Resultado dos artigos selecionados separados por fases ................................... 20

Figura 2 - Solicitação de conexão do dispositivo A ao B ................................................... 35

Figura 3 - Dispositivo B exibe notificação de solicitação de conexão do dispositivo A ao

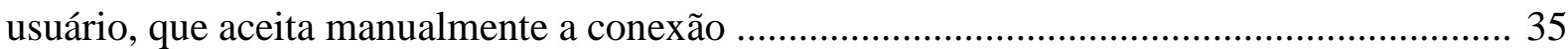

Figura 4 - Dispositivo B envia confirmação do aceite da conexão ao dispositivo A ............. 36

Figura 5 - Dispositivo A realiza um broadcast com as informações da conexão para todas

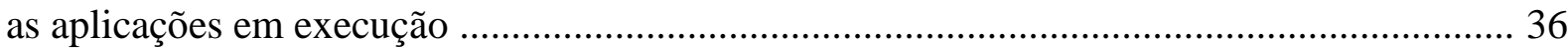

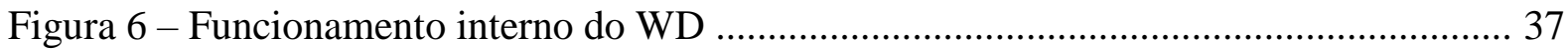

Figura 7 - Solicitação de conexão pelo dispositivo A (a) ao dispositivo B (b) ..................... 37

Figura 8 - Conceito de conexão abordado atualmente ...................................................... 39

Figura 9 - Conceito de conexão e transferência de dados proposto ..................................... 40

Figura 10 - Fluxo atual, simplificado, da solicitação de dispositivos próximos e conexão ... 44

Figura 11 - Fluxo proposto, simplificado, da solicitação de dispositivos próximos e conexão

Figura 12 - Aplicação Together com a conexão entre os dispositivos A e B realizada de forma espontânea

Figura 13 - Lista de arquivos do Contêiner com a porcentagem de dados transferidos de arquivos oriundos de outros dispositivos 


\section{Lista de tabelas}

Tabela 1 - Bancos de dados científicos utilizados ......................................................... 18

Tabela 2 - Strings de pesquisa utilizada por base de dados científica.................................. 19 


\section{Lista de gráficos}

Gráfico 1 - Artigos por categoria, um artigo pode estar em mais de uma categoria ............. 27

Gráfico 2 - Problemas ou limitações encontradas .............................................................. 27

Gráfico 3 - Artigos que encontraram problemas ou limitações e sugerem melhorias ............28 


\section{Lista de quadros}

Quadro 1 - Artigos selecionados 


\section{Lista de abreviaturas e siglas}

ACM Association for Computing Machinery

AP Access Point

API Application Programming Interface

CAPTURE Cooperatively Applied Positioning Techniques Utilizing Range Extension

D2D Device-to-device

DoS Denial-of-Service

EB Exabytes

GB Gigabytes

GO Group Owner

IEEE Institute of Electrical and Eletronic Engineers

IP Internet Protocol

IoT Internet of Things

MAC Media Access Control

NFC Near Field Communication

P2P Peer-to-peer

RSSI Received Signal Strength Indicator

SC Spontaneous Connection

SD ScienceDirect

SSD Solid State Drive

WD Wi-Fi Direct

WDSC Wi-Fi Direct with Spontaneous Connection 


\section{Sumário}

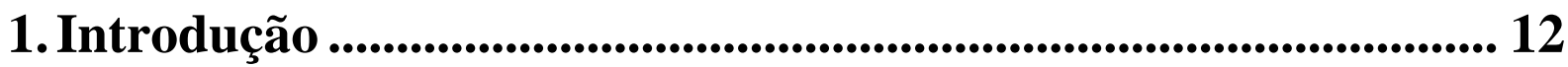

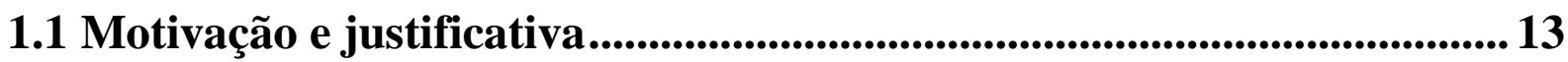

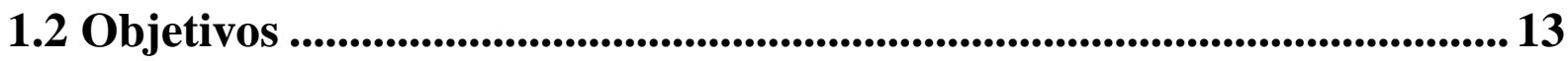

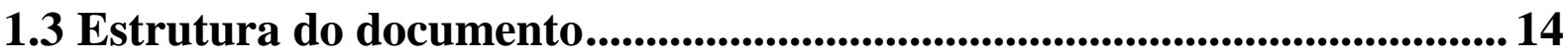

2. Metodologia .................................................................................................... 15

3. Wi-Fi Direct - Aplicações e limitações em dispositivos móveis:

uma Revisão Sistemática ............................................................ 16

3.1 Introduçãa...................................................................................................................... 17

3.2 Métodos .................................................................................................................. 18

3.2.1 Critérios de inclusão e exclusão............................................................ 18

3.2.2 Estratégia de pesquisa ............................................................................................ 19

3.2.3 Procedimentos da revisão........................................................................ 19

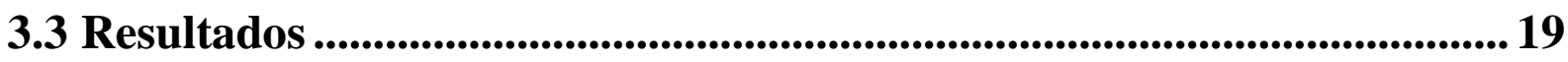

3.3.1 Estatísticas ................................................................................................................. 27

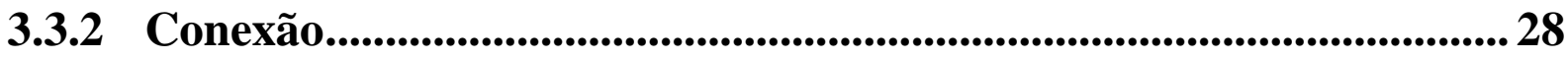

3.3.3 Energia ....................................................................................................................... 29

3.3.4 Formação de grupo......................................................................................... 29

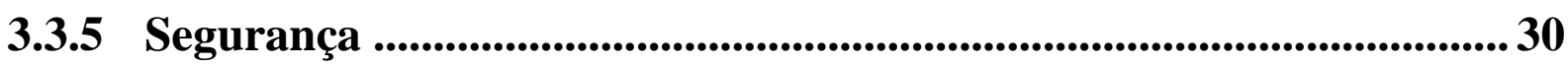

3.4 Discussão ............................................................................................................................... 31

3.5 Considerações finais..................................................................................................... 32

4. Wi-Fi Direct with Spontaneous Connection ................................ 33

4.1 Visão Geral do Wi-Fi Direct no Android.................................................... 34

4.2 Trabalhos Relacionados .....................................................................................38

4.3 Conexão Espontânea..................................................................................................... 39

4.4 Wi-Fi Direct with Spontaneous Connection .............................................. 42 
4.5 Aplicativo com o uso de conexão espontânea - Together 48

4.6 Considerações finais.

5. Conclusões Finais................................................................ 51

Referências.......................................................................................... 53 


\section{Introdução}

O uso de dispositivos móveis no dia a dia das pessoas tem se tornando cada vez mais comum, e a cada dia tem ganhado mais importância. Isso pode ser demonstrado através do crescimento no consumo de dados móveis que dobram a cada ano [1]. Esse crescimento demonstra a importância da Internet no cotidiano das pessoas, que cada vez mais utilizam seus smartphones para tarefas cotidianas. A maioria das atividades realizadas envolvem algum tipo de troca de dados. O que pode ser realizado com o uso da Internet ou de tecnologias como Bluetooth, Near Field Communication (NFC) e Wi-Fi Direct (WD), que permitem que dados sejam transmitidos entre dispositivos próximos.

As tecnologias Bluetooth e WD, utilizadas para realizar conexão com algum dispositivo, requer intervenção manual do usuário para a confirmação de uma conexão, para, só então, liberar a transferência de dados entre os dispositivos envolvidos. Já a comunicação com o uso de NFC não demanda autorização do usuário, pois realiza a conexão por aproximação de dispositivos. Nesse caso, a proximidade dos dispositivos funciona como autorização para conexão. Outra característica da comunicação por NFC é a baixa capacidade para transmissão de dados e baixo alcance. À medida que a distância entre os dispositivos aumenta, a autorização do usuário é requerida para que a conexão seja estabelecida. Essa abordagem visa evitar conexões não desejadas e possíveis problemas de segurança. Entretanto, a solicitação de autorização limita a transferência de dados ao cenário onde os envolvidos na troca de dados estão disponíveis para interagir, um a um, para estabelecer uma conexão. Essa restrição de solicitar autorização prévia para se comunicar dificulta a possibilidade de troca de dados entre vários dispositivos de forma simultânea. Autorizar cada solicitação de conexão se torna uma tarefa exaustiva. Como exemplo, imagine um palestrante que deseje compartilhar com sua plateia um arquivo disponível em seu smartphone. Com os protocolos atuais, todos os presentes necessitariam estabelecer e autorizar a conexão. Por sua vez, o palestrante também necessitaria validá-las. A depender da quantidade de pessoas interessadas no material a ser compartilhado, o tempo e esforço necessários para concluir o compartilhamento poderia inviabilizar essa experiência de troca de dados.

Outros cenários de troca de dados, como compartilhar imagens e vídeos, entre dispositivos próximos também são limitados pela autorização prévia da conexão. Tais exemplos mostram que os dispositivos móveis ainda possuem possibilidades de comunicação e troca de dados a serem exploradas caso surjam novos conceitos e protocolos de comunicação. Para lidar com esse desafio, este trabalho propõe uma alternativa de comunicação entre dispositivos 
próximos, que permite o estabelecimento de conexão sem autorização prévia e que, ainda assim, mantém a capacidade do usuário de decidir se deseja ou não utilizar os dados enviados.

\subsection{Motivação e justificativa}

O crescimento no consumo de dados móveis denota um aumento no uso de dispositivos móveis em todo o planeta. Como consequência, previsões demonstram que esse crescimento contínuo poderá acarretar em uma sobrecarga de dados para as redes móveis e que tecnologias de quarta geração (4G) estão propensas a enfrentar severas restrições de disponibilidade [2]. Esse crescimento no consumo de dados também pode ser justificado pelo aumento da densidade geográfica de dispositivos móveis. O que, por sua vez, poderá transformar a comunicação direta entre dispositivos geograficamente próximos em uma importante alternativa de comunicação.

O potencial para a conexão direta entre dispositivos pode ser percebido, por exemplo, em uma reunião de amigos quando alguém deseja compartilhar arquivos em seu smartphone. Essa tarefa, na grande parte das vezes, acontece com o uso da Internet, ao invés de uso de conexões entre dispositivos, como por Bluetooth. Ou seja, mesmo que os dispositivos estejam lado a lado, os dados compartilhados trafegam pela Internet em um caminho de ida e volta que poderia ser evitado. Em parte, esse processo acontece porque a conexão entre dispositivos é mais complexa do que o envio dos dados pela Internet. Assim, a necessidade de realizar vários passos para estabelecer conexões e até mesmo a falta de conhecimento sobre como realizar a comunicação entre dispositivos limita o seu uso.

No caso dos IoT's - Internet das coisas, do inglês Internet of Things, a necessidade de autorização por uma caixa de diálogo exibida ao usuário é um impeditivo para que dispositivos possam trocar dados de forma simplificada. Tornar a conexão direta um processo tão simples quanto o envio de um arquivo pela Internet, mais do que prover comodidade para interação entre dispositivos próximos, pode ampliar o seu uso em cenários de desconexão com a Internet, proporcionar economia de tráfego de dados e consequente redução de custo com a Internet [3].

\subsection{Objetivos}

O principal objetivo deste trabalho é propor e implementar uma alternativa para comunicação entre dispositivos próximos que não demande autorização manual prévia a cada 
conexão com um dispositivo, e que, ainda assim, permita que o usuário decida se quer ou não usar os dados transmitidos em uma conexão.

Para tanto, os seguintes objetivos específicos foram propostos:

- Revisão sistemática para avaliar as características e limitações de comunicação entre dispositivos móveis;

- Definição de uma arquitetura que possibilite a conexão e transferência de dados entre dispositivos próximos sem a necessidade de intervenção prévia do usuário; e

- Definição e implementação do conceito de comunicação proposto no sistema operacional de código aberto para dispositivos móveis Android.

\subsection{Estrutura do documento}

Este documento está organizado da seguinte forma: capítulo 2 apresenta o método de pesquisa empregado para a realização do trabalho; capítulo 3 apresenta a revisão sistemática da literatura realizada, capítulo 4 apresenta a abordagem concebida para expandir os cenários de utilização da tecnologia; capítulo 5, apresenta as conclusões desta dissertação. 


\section{Metodologia}

A realização deste trabalho foi guiada pela seguinte metodologia. Inicialmente foi realizada uma revisão sistemática da literatura para avaliar as características e limitações da comunicação entre dispositivos móveis. A revisão sistemática também foi utilizada para apoiar a escolha do protocolo a ser estendido com a proposta deste trabalho. A condução da revisão sistemática foi baseada no processo estabelecido por [4], que define três fases para sua realização: (i) planejamento, (ii) condução e (iii) disseminações dos resultados. Na fase de planejamento, a estratégia de busca em base de dados de artigos para identificar estudos primários foi definida, assim como os critérios para seleção dos artigos. Em seguida, a busca e seleção dos artigos foram executadas seguindo o protocolo definido. Nesta revisão sistemática, ambas as etapas foram realizadas por um dos autores e revisadas pelo outro, tendo sido utilizada a ferramenta StArt [5] para auxiliar na organização e na execução dessa revisão sistemática.

A partir da revisão realizada, foram identificadas convergências em relação às limitações do protocolo de comunicação WD e as características do conceito de comunicação proposto por este trabalho. O sistema operacional Android foi escolhido por possuir código aberto e por oferecer uma implementação do WD. Em seguida foram definidos os requisitos para a criação da extensão do protocolo WD para incorporação de nossa proposta de comunicação. Por fim, foi criado uma aplicação para Android para demonstrar o impacto da proposta no desenvolvimento de aplicações e como seus recursos podem ser utilizados. Para o desenvolvimento da extensão do WD foi utilizado o Android em sua versão 6. Os testes foram realizados em 2 smartphones LG Nexus 5 e 1 tablet Samsung Galaxy Tab S. 


\section{Wi-Fi Direct - Aplicações e limitações em dispositivos móveis: uma Revisão Sistemática}

Contexto: De acordo com estudo realizado em 2015 pela Cisco ao final de 2014 o número de dispositivos móveis conectados iria superar o número de habitantes da Terra, e até o final de 2019, estarão previstos 11.5 bilhões de dispositivos móveis conectados [6], o que leva ao aumento correspondente no tráfego de dados móveis. Em 2013 e 2014, houve um aumento de $69 \%$ no tráfego de dados móveis, chegando a 2.5 Exabytes (EB) por mês no final de 2014 e, estima-se, a 24.3 EB por mês até o final de 2019 [6]. Propostas de utilização de tecnologias que permitem conexão direta são vistas como alternativas para a redução do tráfego de dados nas redes de celulares e podem proporcionar comunicação entre dispositivos em áreas onde a Internet não esteja disponível, sendo o protocolo Wi-Fi Direct (WD) uma dessas tecnologias. Criado pela Wi-Fi Alliance, o WD permite conexão direta entre dispositivos sem a necessidade de uma infraestrutura de rede pré-estabelecida.

Objetivo: Identificar formas de uso do WD em aplicativos móveis e pesquisas que visem a sua evolução.

Método: Uma revisão sistemática da literatura foi realizada para analisar as abordagens utilizadas na implementação do WD em dispositivos móveis. Os bancos de dados científicos Association for Computing Machinery (ACM), Institute of Electrical and Eletronic Engineers (IEEE), ScienceDirect (SD) e Scopus foram analisados por meio de uma predeterminada string de pesquisa para identificar os estudos primários, e foram selecionados aqueles que atenderam aos critérios de inclusão e exclusão.

Resultados: De 553 artigos analisados foram selecionados 28 artigos por meio dos critérios de inclusão e exclusão. Desses estudos 20 relatam limitações ou problemas encontrados na utilização do WD, e os 8 restantes utilizam o WD apenas como um meio de comunicação para transmissão de alguma informação.

Discussão e conclusão: A análise dos artigos revelou limitações comumente relatadas que indicam direções para a evolução do protocolo de comunicação WD. Foram identificados problemas e limitações no processo de conexão, formação de grupos, segurança e consumo de energia. Tais problemas e limitações causam barreiras à adesão da tecnologia WD por desenvolvedores e usuários, que fazem opção por desenvolver seus próprios algoritmos de comunicação para lidar com as limitações e problemas do WD. Esse cenário apresenta a necessidade da evolução do protocolo WD ou mesmo o surgimento de novos protocolos que tornem a comunicação direta entre dispositivos móveis e geograficamente próximos uma 
alternativa importante para a troca de informação entre os dispositivos sem a necessidade de Internet.

\subsection{Introdução}

Em um estudo realizado pela Cisco [6], foi estimado que o número de dispositivos móveis conectados ao final de 2014 chegaria a 7.4 bilhões, superando o número de habitantes da Terra. O mesmo estudo previa que, ao final de 2019, esse número chegaria a 11.5 bilhões. Esse aumento na utilização de dispositivos móveis leva ao crescimento correspondente no tráfego de dados móveis. Estima-se que a quantidade de tráfego de dados móveis atingiu 2.5 EB por mês em 2014, e poderá atingir 24.3 EB por mês até o final de 2019 [6]. Esse crescimento implica uma maior utilização das redes de celulares e também amplia a possibilidade de que mais de um dispositivo móvel esteja geograficamente próximo, e que seus proprietários ou aplicativos neles instalados possam ser beneficiados pela comunicação direta entre dispositivos. Entretanto, acredita-se que esse crescimento possa trazer uma sobrecarga de dados às redes de celulares, sendo que mesmo tecnologias de quarta geração (4G) são propensas a enfrentar graves problemas de disponibilidade [2].

Propostas de utilização de tecnologias que permitam conexões diretas entre dispositivos móveis são vistas como alternativas para a redução do tráfego de dados nas redes de celulares [3] e podem proporcionar comunicação em áreas onde a Internet não esteja disponível ou onde um grupo de dispositivos ou pessoas desejem comunicar-se mutuamente. Uma dessas tecnologias é o WD, criado pela Wi-Fi Alliance [7]. O protocolo WD permite a conexão direta entre dispositivos sem a necessidade de um Access Point (AP) e, recentemente, as plataformas móveis Android e Windows Phone passaram a suportar o WD como mais uma opção para conexão direta entre dispositivos (além do NFC, do Bluetooth e do modo ad-hoc do Wi-Fi). Entretanto, devido à integração recente dessas plataformas móveis com a tecnologia WD, estudos estão sendo realizados afim de analisar a utilização da tecnologia. Limitações e problemas em sua implementação e utilização são relatados, assim como melhorias são propostas. Portanto, para identificar o estado da arte no uso do WD em dispositivos móveis, uma revisão sistemática da literatura foi conduzida e apresentada neste artigo. 


\subsection{Métodos}

Com base no método proposto por [4] esta revisão sistemática está dividida em três fases: planejamento, condução e disseminação dos resultados. Na fase de planejamento, a string de pesquisa, os critérios de inclusão e exclusão, e os bancos de dados científicos para a seleção dos artigos são definidos. Após isso, na fase de condução, a pesquisa é realizada e os artigos são selecionados seguindo os critérios definidos na fase de planejamento. Com a coleta dos artigos realizada, é executada a extração dos dados dos artigos selecionados, e esses dados são apresentados. Foi utilizada a ferramenta StArt [5] para auxiliar na organização e na execução desta revisão sistemática.

\subsubsection{Critérios de inclusão e exclusão}

Especificamente, os seguintes critérios de inclusão foram utilizados:

- Artigos que analisaram ou implementaram alguma solução utilizando o WD em dispositivos móveis.

- Bases de dados científicas consideradas, conforme Tabela 1, apresentada a seguir:

Tabela 1 - Bancos de dados científicos utilizados

\begin{tabular}{ll}
\hline Nome & Endereço eletrônico \\
\hline ACM & http://dl.acm.org/ \\
IEEE & http://ieeexplore.ieee.org/ \\
ScienceDirect & http://www.sciencedirect.com/ \\
Scopus & http://www.scopus.com/ \\
\hline
\end{tabular}

Fonte: Ricardo César Corrêa, 2016

Em razão de o WD ser um recente protocolo definido pela Wi-Fi Alliance, não houve restrições quanto à data de publicação dos artigos selecionados, sendo definido como critério de exclusão os artigos que não descrevem a forma como o WD foi utilizado. 


\subsubsection{Estratégia de pesquisa}

Os artigos foram selecionados a partir das bases de dados científicas apresentadas na tabela 1 em 26/12/2015 a partir das strings de pesquisa. Sinônimos do WD foram utilizados para a formação das strings de pesquisa apresentadas na Tabela 2:

Tabela 2 - Strings de pesquisa utilizada por base de dados científica.

\begin{tabular}{ll}
\hline Nome & Endereço eletrônico \\
\hline \multirow{2}{*}{ ACM } & "wi-fi direct" "wifi direct" "wifi-direct" "wi-fi p2p" "wifi p2p" "wifi- \\
& p2p" \\
& "wi-fi direct" OR "wifi direct" OR "wifi-direct" OR "wi-fi p2p" OR \\
IEEE & "wifi p2p" OR "wifi-p2p" \\
& "wi-fi direct" OR "wifi direct" OR "wifi-direct" OR "wi-fi p2p" OR \\
ScienceDirect & "wifi p2p" OR "wifi-p2p" \\
& "wi-fi direct" OR "wifi direct" OR "wifi-direct" OR "wi-fi p2p" OR \\
Scopus & "wifi p2p" OR "wifi-p2p" \\
\hline
\end{tabular}

Fonte: Ricardo César Corrêa, 2016

\subsubsection{Procedimentos da revisão}

Os artigos foram selecionados de acordo com o critério de inclusão e exclusão por meio da leitura do título e do abstract. Os artigos selecionados foram lidos na íntegra para a fase de extração de dados. Sendo extraídos os seguintes dados:

- Autores

- Título

- Resumo

- Ano Publicação

- Categoria (s)

- Limitação(ões)/Problema(s)s

\subsection{Resultados}

Nas pesquisas realizadas nas bases científicas, foram encontrados 553 artigos, 135 duplicados. Durante a fase de seleção, após a leitura do título e do resumo de cada um, foram 
selecionados 164 artigos e 254 foram rejeitados. Durante a fase de extração, os artigos selecionados na fase anterior foram lidos integralmente, e 28 deles foram incluídos na revisão sistemática. Na figura a seguir, são apresentados os estágios de identificação, seleção e extração dos artigos.

Figura 1 - Resultado dos artigos selecionados separados por fases

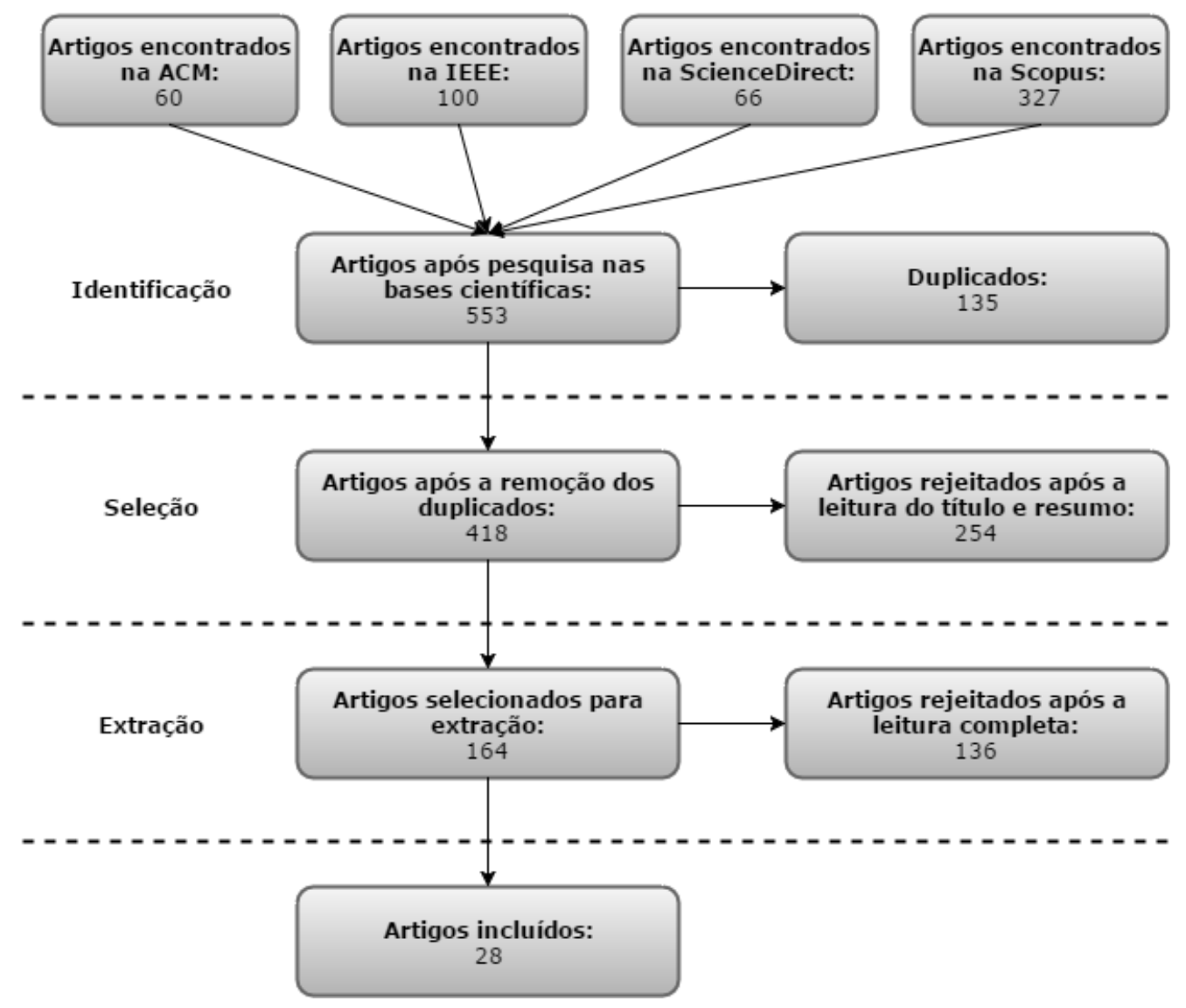

Fonte: Ricardo César Corrêa, 2016

Após a seleção dos artigos, foi realizada a extração de dados apresentados na Figura 1. A partir dessa extração, é possível determinar o estado da arte na utilização do WD em dispositivos móveis, identificando em que o WD tem sido utilizado, quais os problemas encontrados e quais as melhorias propostas. A partir dessa extração, foi possível descobrir a área de foco na utilização do WD em cada artigo selecionado, realizando uma categorização de cada estudo para auxiliar na discussão dos resultados obtidos. Essas categorias foram divididas em Conexão, Energia, Formação de Grupo e Segurança, sendo classificadas em uma dessas áreas, e apresentados os dados no Quadro 1, conforme descrito a seguir:

- Conexão: estudos que atuaram na fase de descoberta do WD. 
- Energia: estudos que avaliaram o consumo de energia do WD.

- Formação de Grupo: estudos que atuaram no estabelecimento de conexão entre múltiplos dispositivos ou em dispositivos em outros grupos.

- Segurança: estudos que avaliaram as vulnerabilidades da implementação do WD. 
Quadro 1 - Artigos selecionados

\begin{tabular}{|c|c|c|c|c|c|c|}
\hline & Autores & Categoria(s) & Título & Resumo & $\begin{array}{l}\text { Limitação(ões)/ } \\
\text { Problema(s) }\end{array}$ & $\begin{array}{l}\text { Ano } \\
\text { Publicação }\end{array}$ \\
\hline [8] & $\begin{array}{l}\text { Ahmed A. Shahin } \\
\text { Mohamed Younis }\end{array}$ & $\begin{array}{l}\text { Conexão } \\
\text { Formação de } \\
\text { Grupo }\end{array}$ & $\begin{array}{l}\text { A Framework for P2P } \\
\text { Networking of Smart Devices } \\
\text { Using Wi-Fi Direct }\end{array}$ & $\begin{array}{l}\text { Apresenta um framework para formação e o gerenciamento de } \\
\text { grupos de comunicação na utilização do WD. }\end{array}$ & $\begin{array}{l}\text { Obtenção e } \\
\text { distribuição do } \\
\text { Internet Protocol } \\
\text { (IP) de cada } \\
\text { dispositivo. }\end{array}$ & 2014 \\
\hline [9] & $\begin{array}{l}\text { Le Van Hoang } \\
\text { Hitoshi Ogawa }\end{array}$ & Conexão & $\begin{array}{l}\text { A platform for building ad hoc } \\
\text { social networks based on Wi-Fi } \\
\text { direct }\end{array}$ & $\begin{array}{l}\text { Propõe uma plataforma para construção de redes ad hoc, } \\
\text { utilizando WD. }\end{array}$ & $\begin{array}{l}\text { Intervenção do } \\
\text { usuário para } \\
\text { realização de uma } \\
\text { conexão. }\end{array}$ & 2014 \\
\hline [10] & $\begin{array}{l}\text { Ari Hadiks } \\
\text { et al. }\end{array}$ & Segurança & $\begin{array}{l}\text { A Study of Stealthy Denial-of- } \\
\text { Service Attacks in Wi-Fi Direct } \\
\text { Device-to-Device Networks }\end{array}$ & $\begin{array}{l}\text { Explora os possíveis problemas de segurança em uma rede WD } \\
\text { com o foco em ataques de Denial-of-Service (DoS). Conclui que } \\
\text { usuários mal-intencionados podem privar a capacidade de usuários } \\
\text { inocentes desfrutarem da conveniência dada pela rede formada } \\
\text { entre dispositivos. }\end{array}$ & $\begin{array}{l}\text { Possibilidade de } \\
\text { ataque DoS. }\end{array}$ & 2014 \\
\hline [11] & $\begin{array}{l}\text { Yufeng Wang } \\
\text { et al. }\end{array}$ & Conexão & $\begin{array}{l}\text { A Wi-Fi Direct based P2P } \\
\text { application prototype for } \\
\text { mobile social networking in } \\
\text { proximity (MSNP) }\end{array}$ & $\begin{array}{l}\text { Propõe uma rede peer-to-peer (P2P) a qual permite a troca de } \\
\text { dados entre dispositivos móveis sem a necessidade de uma } \\
\text { infraestrutura de rede por meio da utilização do WD, } \\
\text { desenvolvendo uma solução para descoberta e privacidade dos } \\
\text { dispositivos da rede. }\end{array}$ & Nenhuma. & 2014 \\
\hline [12] & $\begin{array}{l}\text { Sacha Trifunovic } \\
\text { et al. }\end{array}$ & Energia & $\begin{array}{l}\text { Adaptive Role Switching for } \\
\text { Fair and Efficient Battery } \\
\text { Usage in Device-to-device } \\
\text { Communication }\end{array}$ & $\begin{array}{l}\text { Apresenta medições do consumo de energia na comunicação entre } \\
\text { dispositivos móveis com as tecnologias Bluetooth, WD e WLAN. } \\
\text { Demonstra que todas as comunicações entre dispositivos sofrem } \\
\text { de dois grandes problemas no consumo de energia: descoberta de } \\
\text { vizinhos e manutenção da conexão realizada de forma injusta. } \\
\text { Para tornar o consumo de energia justo na manutenção da conexão } \\
\text { propõe um esquema de troca de papéis entre os dispositivos, } \\
\text { equilibrando o consumo. }\end{array}$ & $\begin{array}{l}\text { Consumo de } \\
\text { energia injusto } \\
\text { entre o Group } \\
\text { Owner (GO) e os } \\
\text { membros do grupo. }\end{array}$ & 2014 \\
\hline
\end{tabular}




\begin{tabular}{|c|c|c|c|c|c|c|}
\hline [13] & $\begin{array}{l}\text { Paul Wong } \\
\text { et al. }\end{array}$ & $\begin{array}{l}\text { Conexão } \\
\text { Formação de } \\
\text { Grupo }\end{array}$ & $\begin{array}{l}\text { Automatic Android-based } \\
\text { wireless mesh networks }\end{array}$ & $\begin{array}{l}\text { Contorna as limitações do WD de falta de apoio para formação de } \\
\text { redes muitos-para-um e no estabelecimento de conexão, da qual } \\
\text { requer interação do usuário para sua realização. }\end{array}$ & $\begin{array}{l}\text { Intervenção do } \\
\text { usuário para } \\
\text { realização de uma } \\
\text { conexão. }\end{array}$ & 2014 \\
\hline [14] & Gary Cullen et al. & $\begin{array}{l}\text { Conexão } \\
\text { Energia }\end{array}$ & $\begin{array}{l}\text { CAPTURE - Extending the } \\
\text { Scope of Self-Localization in } \\
\text { Indoor Positioning Systems }\end{array}$ & $\begin{array}{l}\text { O artigo apresenta o framework "Cooperatively Applied } \\
\text { Positioning Techniques Utilizing Range Extension" (CAPTURE) } \\
\text { que utiliza a informação Received Signal Strength Indicator } \\
\text { (RSSI) fornecida pelo WD para detectar a localização de um } \\
\text { dispositivo em um ambiente interno. }\end{array}$ & Nenhuma. & 2015 \\
\hline$[15]$ & $\begin{array}{l}\text { Claudio Casetti } \\
\text { et al. }\end{array}$ & $\begin{array}{l}\text { Formação de } \\
\text { Grupo }\end{array}$ & $\begin{array}{l}\text { Content-centric Routing in Wi- } \\
\text { Fi Direct Multi-group } \\
\text { Networks }\end{array}$ & $\begin{array}{l}\text { Aborda e implementa roteamento de conteúdo em uma arquitetura } \\
\text { P2P utilizando WD. É desenvolvido uma comunicação } \\
\text { bidirecional e multi-grupo para superar a limitação da topologia de } \\
\text { rede e o endereçamento do WD. }\end{array}$ & $\begin{array}{l}\text { Obtenção e } \\
\text { distribuição do IP } \\
\text { de cada dispositivo. }\end{array}$ & 2015 \\
\hline & & & & & Topologia de rede. & \\
\hline$[16]$ & Igor Bisio et al. & $\begin{array}{l}\text { Formação de } \\
\text { Grupo }\end{array}$ & $\begin{array}{l}\text { Context-awareness over } \\
\text { transient cloud in D2D } \\
\text { networks: energy performance } \\
\text { analysis and evaluation }\end{array}$ & $\begin{array}{l}\text { Apresenta um contexto de computação colaborativa que permite } \\
\text { dispositivos próximos formar uma rede ad hoc, a partir do WD, } \\
\text { para distribuição de tarefas de baixo nível, a fim de poupar } \\
\text { recursos de CPU e energia de um dispositivo móvel. }\end{array}$ & Nenhuma. & 2015 \\
\hline [17] & $\begin{array}{l}\text { Nikki Broch } \\
\text { Ashton } \\
\text { et al. }\end{array}$ & Conexão & $\begin{array}{l}\text { Cool-SHARE: Offload } \\
\text { Smartphone Data by Sharing }\end{array}$ & $\begin{array}{l}\text { O artigo apresenta a implementação do Cool-SHARE para } \\
\text { compartilhamento de aplicativos utilizando WD. Contorna a } \\
\text { limitação de necessidade de intervenção do usuário para conexão e } \\
\text { realiza uma análise de performance do WD para as fases de } \\
\text { descoberta e conexão. A partir dos dados coletados propõe } \\
\text { melhorias para a redução do tempo de conexão. }\end{array}$ & $\begin{array}{l}\text { Intervenção do } \\
\text { usuário para } \\
\text { realização de uma } \\
\text { conexão. } \\
\text { Repetição da } \\
\text { transmissão de } \\
\text { pacotes } \\
\text { desnecessários para } \\
\text { o estabelecimento } \\
\text { de uma conexão. }\end{array}$ & 2014 \\
\hline [18] & $\begin{array}{l}\text { Chao Yao } \\
\text { et al. }\end{array}$ & $\begin{array}{l}\text { Conexão } \\
\text { Formação de } \\
\text { Grupo }\end{array}$ & $\begin{array}{l}\text { Demo: WiFi Multihop - } \\
\text { Implementing Device-to- } \\
\text { Device Local Area Networks } \\
\text { by Android Smartphones }\end{array}$ & $\begin{array}{l}\text { Propõe uma extensão para a implementação do WD permitir a } \\
\text { comunicação multi-grupo e a eleição do líder com base em } \\
\text { topologia e energia. }\end{array}$ & $\begin{array}{l}\text { Eleição do líder } \\
(\mathrm{GO}) .\end{array}$ & 2015 \\
\hline
\end{tabular}


[19] Jae Yoon Jung Conexão Dong Kwan Kim

\section{Formação de}

Grupo

[20] Urbano B

et al.

[21] Marco Conti et al.

Formação de Grupo

[22] I Hsuan Peng et Formação de al.

Grupo

[23] Ricardo P. Marinh et al.

Formação de Grupo

[24] Matteo Poza et al.

Formação de

Grupo

[25] Hongxu Zhang et al.
Development of an Instant

Meeting Android Application

Using Wi-Fi Direct APIs

Dynamic Clustering in WiFi Direct Technology

Experimenting opportunistic networks with WiFi Direct

Implementation of Resource Integration and Application of Grouped Mobile Devices Using Wi-Fi Direct

IMSN Routing on Wi-Fi Direct Enabled Devices

Mobile Data Offloading: An Experimental Evaluation

mQual: A Mobile Peer-to-Pee Network Framework Supporting Quality of Service
O trabalho apresenta uma aplicação para realização de reuniões

utilizando o WD: por meio dessa aplicação, o apresentador

distribui um documento entre os participantes e suas marcações mudança de página e zoom são transmitidos e reproduzidos nos dispositivos conectados.

Introduz o conceito de clusterização em redes WD. A partir dessa clusterização a aplicação proposta troca informações entre os dispositivos para a eleição do líder (GO) mais adequado ao grupo do WD.

Investiga a viabilidade de criação de redes oportunistas utilizando WD por meio da análise de performance do protocolo em cenário reais com um número variável de dispositivos, concluindo que o modo autônomo possui melhor performance para a formação do grupo.

O trabalho apresenta uma aplicação que permite a integração da exibição de múltiplas telas de dispositivos em uma única

visualização, utilizando NFC e WD para a troca de dados entre os dispositivos.

Demonstra a aplicação de 4 algoritmos de roteamento sobre o WD, modificando-o para permitir comunicação multi-hop e apresentando o algoritmo mais adequado. A partir dos resultados, desenvolve um algoritmo para roteamento de dados utilizando WD.

Descreve a aplicação BlueFall para testes de protocolos swarming Nenhuma. usando dispositivos móveis, demonstrando dois esquemas de swarming utilizando Bluetooth e WD para demonstrar a utilização da aplicação para testes de protocolos swarming.

$\mathrm{O}$ artigo apresenta o framework mQual para criação e manutenção de redes entre dispositivos móveis, complementando a

Nenhuma.

leição do líder

Nenhuma.

Topologia de rede. intensidade do sinal. 


\begin{tabular}{|c|c|c|c|c|c|c|}
\hline$[26]$ & $\begin{array}{l}\text { Bhojan Anand } \\
\text { Tan Guo Wei }\end{array}$ & Conexão & $\begin{array}{l}\text { mumble - Framework for } \\
\text { Seamless Message Transfer on } \\
\text { Smartphones }\end{array}$ & $\begin{array}{l}\text { Demonstra a possibilidade de transferência de dados utilizando } \\
\text { WD sem a necessidade de pareamento, autenticação, acesso root } \\
\text { ou conectividade com a Internet. Por meio do serviço de } \\
\text { descoberta, um método é desenvolvido para permitir essa } \\
\text { transmissão de dados. }\end{array}$ & $\begin{array}{l}\text { Intervenção do } \\
\text { usuário para } \\
\text { realização de uma } \\
\text { conexão. }\end{array}$ & 2013 \\
\hline$[27]$ & $\begin{array}{l}\text { Isaí Michel } \\
\text { Lombera } \\
\text { et al. }\end{array}$ & $\begin{array}{l}\text { Conexão } \\
\text { Formação de } \\
\text { Grupo }\end{array}$ & $\begin{array}{l}\text { Peer-to-peer publication, search } \\
\text { and retrieval using the Android } \\
\text { mobile platform }\end{array}$ & $\begin{array}{l}\text { Apresenta a aplicação iTrust para publicação, procura e } \\
\text { recuperação de dados utilizando o WD, descrevendo sua solução } \\
\text { para contornar a limitação do WD de associação do endereço } \\
\text { Media Access Control (MAC) com o IP dos dispositivos. }\end{array}$ & $\begin{array}{l}\text { Obtenção e } \\
\text { distribuição do IP } \\
\text { de cada dispositivo. }\end{array}$ & 2014 \\
\hline$[28]$ & $\begin{array}{l}\text { Seokung Yoon } \\
\text { et al. }\end{array}$ & Segurança & $\begin{array}{l}\text { Security Analysis of } \\
\text { Vulnerable Wi-Fi Direct }\end{array}$ & $\begin{array}{l}\text { Demonstra a possibilidade de ataques de deauthentication e key } \\
\text { cracking no WD. }\end{array}$ & $\begin{array}{l}\text { Possibilidade de } \\
\text { ataque } \\
\text { deauthentication } \mathrm{e} \\
\text { key cracking. }\end{array}$ & 2012 \\
\hline [29] & $\begin{array}{l}\text { Moreno } \\
\text { Ambrosin } \\
\text { et al. }\end{array}$ & $\begin{array}{l}\text { Formação de } \\
\text { Grupo }\end{array}$ & $\begin{array}{l}\text { Smartphone and Laptop } \\
\text { Frameworks for Vehicular } \\
\text { Networking Experimentation }\end{array}$ & $\begin{array}{l}\text { Apresenta dois frameworks para possibilitar testes de redes } \\
\text { veiculares, um deles baseado no WD. Na utilização do WD, } \\
\text { contorna a limitação de roteamento. }\end{array}$ & Topologia de rede. & 2013 \\
\hline$[30]$ & $\begin{array}{l}\text { Aurelio Monarrez } \\
\text { et al. }\end{array}$ & $\begin{array}{l}\text { Conexão } \\
\text { Formação de } \\
\text { Grupo }\end{array}$ & $\begin{array}{l}\text { Supporting First-Responders in } \\
\text { Infrastructure-less } \\
\text { Environments }\end{array}$ & $\begin{array}{l}\text { Demonstra uma extensão para o WD que automatiza os processos } \\
\text { de descoberta, conexão e formação de grupo. }\end{array}$ & $\begin{array}{l}\text { Intervenção do } \\
\text { usuário para } \\
\text { realização de uma } \\
\text { conexão. }\end{array}$ & 2015 \\
\hline$[31]$ & $\begin{array}{l}\text { Terry Penner } \\
\text { et al. }\end{array}$ & $\begin{array}{l}\text { Formação de } \\
\text { Grupo }\end{array}$ & $\begin{array}{l}\text { Transient Clouds Assignment } \\
\text { and Collaborative Execution of } \\
\text { Tasks on Mobile Devices }\end{array}$ & $\begin{array}{l}\text { Propõe Transient Clouds, uma plataforma colaborativa para } \\
\text { permitir que dispositivos próximos formem uma rede e forneçam } \\
\text { serviços. A partir disso é disponibilizada uma implementação em } \\
\text { Android utilizando WD, citando a limitação de comunicação entre } \\
\text { grupos. }\end{array}$ & Topologia de rede. & 2015 \\
\hline$[32]$ & $\begin{array}{l}\text { Fabio } \\
\text { Malabocchia } \\
\text { et al. }\end{array}$ & $\begin{array}{l}\text { Formação de } \\
\text { Grupo }\end{array}$ & $\begin{array}{l}\text { Using Information Centric } \\
\text { Networking for Mobile } \\
\text { Devices Cooperation at the } \\
\text { Network Edge }\end{array}$ & $\begin{array}{l}\text { Demonstra uma aplicação em Android com WD que explora a } \\
\text { abordagem multi-RAT para streaming de vídeo colaborativo. Por } \\
\text { meio dos testes realizados, relata o problema de sobrecarga de } \\
\text { dados do GO na configuração de topologia estrela, que não se } \\
\text { mantém ligada em razão da sobrecarga de dados. }\end{array}$ & Topologia de rede. & 2015 \\
\hline
\end{tabular}


[33] Hongxu Zhang Conexão et al.

\section{Formação de} Grupo

WD2: An Improved WiFi-
Direct Group Formation
Protocol

[34] Yufeng Duan et al.

Formação de

Grupo

[35] Jingyun Feng et al.

\section{Formação de} Grupo Safety

Wireless Channel Loss
Aborda as limitações no WD da falta de clareza na documentação para a formação de grupos com mais de dois dispositivos e período de escuta do serviço de descoberta desconhecido. Para contornar essas limitações, propõe o protocolo Wi-Fi Direct 2

(WD2) e realiza a sua implementação no Android, reduzindo o tempo de formação de grupo em até $250 \%$.

Wi-Fi Direct Multi-group Data Propõe e implementa um mecanismo para permitir a comunicação Dissemination for Public entre dispositivos que estejam em grupos diferentes na utilização do WD.

Realiza experimentos para analisar a performance de modelos propostos na literatura nos canais de comunicação do WD. É proposto e validado um novo modelo baseado no Gilbert-Elliot provando que o novo modelo supera os outros avaliados.

Obtenção e

distribuição do IP

Analysis - A Case Study Using WiFi-Direct de cada dispositivo.

Eleição do líder (GO).

Topologia de rede.

Nenhuma.

Fonte: Ricardo César Corrêa, 2016 


\subsubsection{Estatísticas}

O gráfico 1 apresenta a quantidade de artigos por categoria, sendo que um artigo pode estar em mais de uma categoria; no gráfico 2, a quantidade daqueles que relatam problemas ou limitações encontradas; e, no gráfico 3, os artigos que apresentam problemas ou limitações, e sugeriram propostas de melhorias. Após a apresentação desses dados são descritos os problemas, as limitações e as propostas realizadas por categoria.

Gráfico 1 - Artigos por categoria, um artigo pode estar em mais de uma categoria

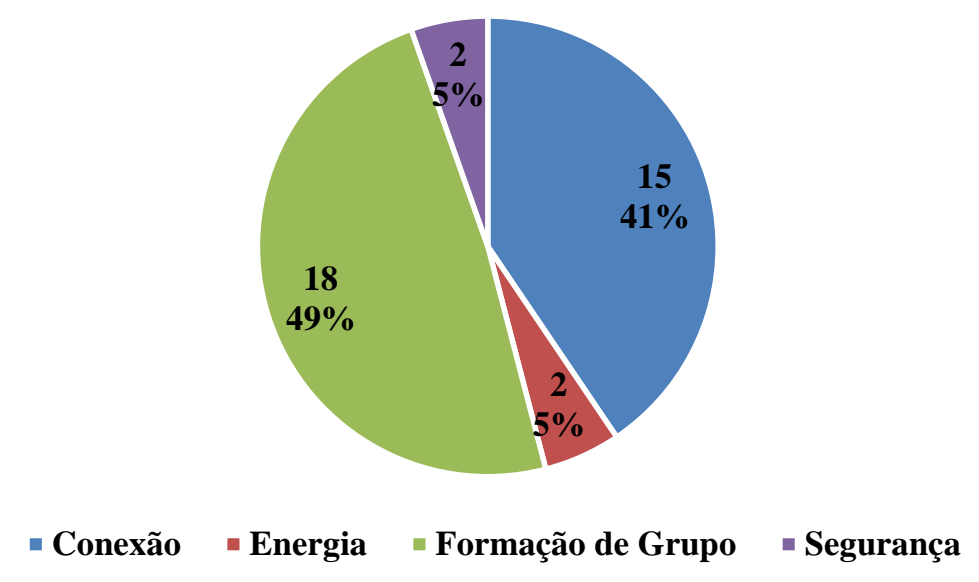

Fonte: Ricardo César Corrêa, 2016

Gráfico 2 - Problemas ou limitações encontradas

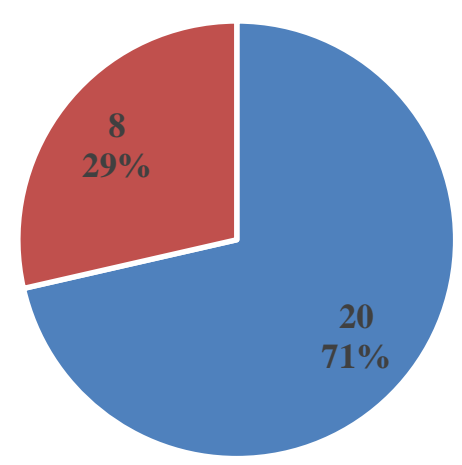

- Relata problemas ou limitações - Não relata nenhum problema ou limitação 
Gráfico 3 - Artigos que encontraram problemas ou limitações e sugerem melhorias

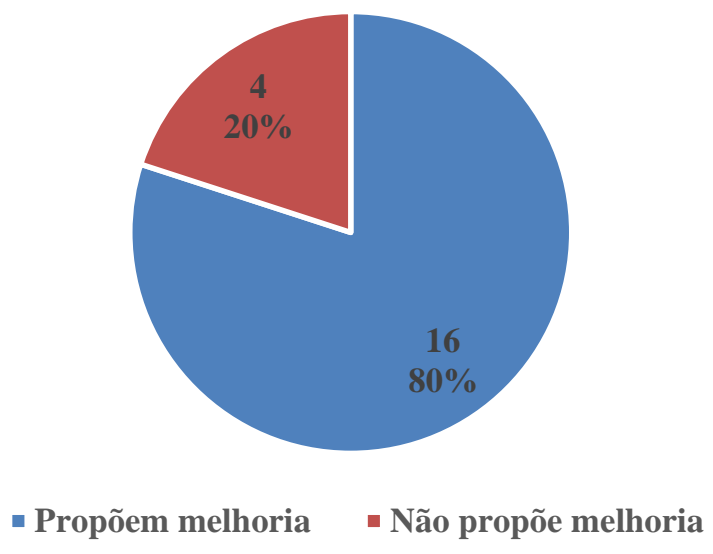

Fonte: Ricardo César Corrêa, 2016

\subsubsection{Conexão}

Para a realização de conexão entre os dispositivos móveis foram descritas as seguintes limitações:

- Associação do IP com o endereço MAC: a atual implementação do WD no Android não possui uma associação direta do endereço MAC com o IP, sendo necessário complementar a implementação para realizar esse controle. Os artigos [8] e [27] contornam essa limitação descrevendo e implementando meios de controle para essa associação e expõem a necessidade de desenvolver operações próprias para a obtenção do IP do dispositivo da interface de rede do WD, pois não existe operação documentada para obter essa informação.

- Eleição do líder (GO): para determinar o GO, é utilizado um valor de intenção, quanto maior esse número, maior é a intenção do dispositivo de se tornar o GO. Em [33], é descrito que, no Android, esse número está fixo em 6. Os trabalhos [18], [20], [25] e [33] descrevem e implementam mecanismos para a eleição do líder do grupo (GO) mais adequado, considerando fatores como intensidade do sinal, CPU/RAM e tempo da bateria, assim contornando essa limitação.

- Intervenção do usuário para iniciar a descoberta e confirmar uma conexão: o WD no Android necessita da intervenção do usuário para iniciar o processo de descoberta e confirmação manual em uma solicitação de conexão. Os trabalhos [9], 
[13], [17], [26] e [30] descrevem e desenvolvem mecanismos para automatizar esses processos.

\subsubsection{Energia}

Para o consumo de energia foram encontrados os problemas a seguir apresentados:

- Fase de descoberta com alto consumo de energia: o trabalho [12] relata que a fase de descoberta de dispositivos possui alto consumo de energia se comparadas com Bluetooth e WLAN, porém não propõe melhorias.

- Manutenção da conexão injusta: no trabalho [12], as operações foram medidas de forma isolada para identificação do consumo de energia de cada fase do WD, detectando que o consumo da manutenção de uma conexão já estabelecida é desproporcional entre o GO e os membros do grupo, fazendo que o GO consuma até 5 vezes mais energia que os outros dispositivos. Para contornar esse problema e tornar o consumo de energia justo [12], cria um mecanismo para a realização da troca de papéis, na qual cada dispositivo da rede passa a ter o papel do GO por um período de tempo.

\subsubsection{Formação de grupo}

Para a formação de grupos foram encontradas as limitações:

- Distribuição dos IPs de cada membro do grupo: para permitir uma rede P2P é necessário que cada dispositivo de uma rede possa identificar o IP dos outros dispositivos, a fim de que, assim, tente estabelecer uma conexão direta. A atual versão do WD no Android não oferece essa funcionalidade. Consequentemente, fica a cargo do desenvolvedor criar tais controles. Nos trabalhos [8], [27] e [33], são realizadas implementações para amenizar o problema de distribuição dos IPs pelo GO para todos os membros do grupo.

- Topologia: o WD no Android permite apenas a formação de uma rede em topologia estrela, na qual todos os membros se conectam ao GO e, a partir dele, comunicamse entre si. Também não permite a comunicação entre dispositivos que estejam em diferentes grupos, como citado por [31]. A topologia de rede em estrela traz alguns problemas para o GO. Nos testes realizados em [32], devido à quantidade de dados trafegados, o GO não suportou e ficou off-line, quebrando a conexão com todos os 
membros do grupo. Em [15], [18], [23] e [34] é desenvolvida uma comunicação multi-grupo, contornando a limitação do WD para comunicação entre dispositivos que estejam em diferentes grupos e, em [29], é realizada uma modificação para permitir o roteamento automático de dados entre dispositivos, entretanto, pode ocorrer o mesmo problema relatado por [32] e o GO pode ficar off-line.

\subsubsection{Segurança}

As análises de segurança encontraram as seguintes limitações:

- Possibilidade de desconectar um membro de um grupo: o trabalho [10] relata a possibilidade de usuários mal-intencionados privarem a capacidade dos usuários desprevenidos de desfrutarem da conveniência dada pela rede device-to-device (D2D). Por meio de experimentos, demonstra um ataque DoS contra os membros de um mesmo grupo, derrubando a conexão de alguns dispositivos. Em [28], é relatada e demonstrada na prática a possibilidade do ataque de deauthentication. Nesse ataque, o agressor captura o endereço MAC do dispositivo-alvo e, a partir deste, consegue quebrar uma conexão entre dispositivos em uma rede WD. Em ambos os estudos, [10] e [28], não há proposta de nenhuma melhoria.

- Possibilidade de forjar uma conexão: o WD possui mecanismos de segurança WPA2. No caso do WPA2 PSK, é possível realizar o key cracking. O estudo [28] demonstra esse ataque por meio da captura de pacotes (air sniffing) e, com a informação obtida, realiza a tentativa de um ataque de dicionário, assim descobrindo a chave necessária para se conectar ao grupo de dispositivos sem permissão. 


\subsection{Discussão}

A partir dos estudos analisados, é possível perceber diversas limitações e problemas encontrados na utilização da implementação do WD no Android. Parte dos estudos contornam as limitações e os problemas encontrados, apresentando soluções para contextos das próprias aplicações.

Em [8] e [27], é descrita a implementação de mecanismos para associar o endereço MAC ao endereço IP, entretanto, solução semelhante poderia ser incorporada à implementação do WD, para que a identificação do IP do endereço MAC em ambos os dispositivos (GO e o membro do grupo) fosse possível no momento da conexão.

A definição do GO é determinada pelo valor de intenção no momento do estabelecimento da conexão, porém, conforme descrito em [33], esse valor está como uma constante com o valor 6 , forçando a escolha aleatória do dispositivo que terá o papel do GO. Essa limitação impede a escolha do dispositivo ideal para se tornar o GO. As soluções apresentadas por [18], [20], [25] e [33] contornam isso, propondo adaptações. Entretanto, essas adaptações fixam o cálculo de alguma forma, considerando fatores como intensidade do sinal, CPU/RAM disponíveis ou tempo da bateria. Apesar de aumentar a dinâmica de atribuição do valor para a escolha do GO, o protocolo poderia ser aprimorado para permitir que o desenvolvedor escolha seus próprios critérios, apoiar ou gerenciar o critério utilizado para escolha do GO.

Para o estabelecimento de uma conexão, é necessária a intervenção do usuário para sua confirmação. Os trabalhos [9], [13], [17], [26] e [30] apresentam um serviço de descoberta para o WD que oferece os dados necessários para que os dispositivos se conectem entre si sem a necessidade de intervenção do usuário, porém todas as soluções propostas são fornecidas pelo desenvolvedor da aplicação que interfere no serviço de descoberta fornecido pelo sistema operacional, essa troca de dados complementar poderia ser incorporada ao serviço de descoberta do WD reduzindo a necessidade de desenvolvimento de rotinas adicionais.

Em relação ao consumo de energia, é detectado que o WD consome energia de forma injusta entre o GO e os membros do grupo para manter a conexão [12]. Essa diferença evidencia a necessidade de revisão nesse processo. No trabalho [17], mapeamentos realizados pelo estabelecimento de uma conexão mostraram a existência da repetição da transmissão de pacotes desnecessários. Isso implica em um atraso no tempo total para o estabelecimento de uma conexão e consumo de recursos desnecessários. 
A tarefa de formação de grupos demanda que o GO distribua os IPs dos membros do grupo para que os dispositivos possam conectar-se entre si e trocar informações sem a necessidade de sempre se comunicarem com o GO. Uma alternativa para essa abordagem seria fornecer um mecanismo para que os desenvolvedores possam escolher se desejam uma implementação em topologia estrela ou D2D, caso seja D2D, sempre no ingresso de um novo dispositivo no grupo, o IP do novo dispositivo seria distribuído para todos os membros. Em relação à segurança, os trabalhos [10] e [28] relatam a possibilidade de ataques DoS, deauthentication e key cracking, porém não apresentam alternativas para lidar com esses problemas, apenas concluem com a necessidade de novos estudos.

\subsection{Considerações finais}

O crescente mercado dos dispositivos móveis e a constante necessidade de comunicação tornam o WD uma tecnologia que irá complementar as comunicações entre dispositivos. Entretanto, em razão da sua recente implementação em dispositivos móveis, é possível perceber pontos a serem explorados a fim de melhorar sua implementação em dispositivos móveis com Android. Os trabalhos aqui analisados apresentam uma clara possibilidade de evolução do protocolo do WD. Conforme demonstra as limitações em áreas como: estabelecimento de conexão, consumo de energia, formação de grupos e segurança. A falta desses recursos limita a adesão do WD por desenvolvedores. O que limita a criação de aplicações que se beneficiem da comunicação entre dispositivos próximos sem a necessidade de conexão web. 


\section{Wi-Fi Direct with Spontaneous Connection}

O crescimento do número de dispositivos móveis e a popularização de serviços oferecidos pela Internet contribuem para o aumento do tráfego de dados móveis, que alcançou 3.7 exabytes (EB) por mês em 2015 e está previsto que chegue a 30.6 EB por mês até 2020 [35], crescimento de quase 10 vezes. Previsões demonstram que esse crescimento poderá acarretar uma sobrecarga de dados para as redes móveis e que tecnologias de quarta geração (4G) estão propensas a enfrentar severas restrições de disponibilidade [2].

Esse crescimento denota a importância da Internet no contidiano das pessoas e que a comunicação entre dispositivos geograficamente próximos poderá se tornar uma importante alternativa de comunicação, não somente pelo baixo custo, mas também considerando que o aumento da densidade geográfica de dispositivos móveis proporcionará maiores oportunidades de comunicação direta entre dispositivos. Seja para comunicações peer-to-peer $(\mathrm{P} 2 \mathrm{P})$, broadcast, estabelecimento de redes locais e conexões entre Internet of Things (IoT's).

O potencial para a conexão direta entre dispositivos pode ser percebido quando em uma reunião de amigos alguém deseja compartilhar arquivos em seu smartphone. Essa tarefa, na grande parte das vezes, acontece com o uso da Internet, ao invés de uso de conexões entre dispositivos, como por Bluetooth. Ou seja, mesmo que os dispositivos estejam lado a lado, os dados compartilhados trafegam pela Internet em um caminho de ida e volta que poderia ser evitado. Em parte, esse processo acontece, porque a conexão entre dispositivos é mais complexa do que o envio dos dados pela Internet. A necessidade de realizar vários passos para estabelecer conexões e até mesmo a falta de conhecimento sobre como realizar a comunicação entre dispositivos limita o seu uso. No caso dos IoT's, a necessidade de uma autorização por uma caixa de diálogo exibida ao usuário é um impeditivo para que dispositivos possam trocar dados de forma simplificada. Tornar a conexão direta um processo tão simples quanto o envio de um arquivo pela Internet, mais do que prover comodidade para interação entre dispositivos próximos, pode ampliar o seu uso em cenários de desconexão com a Internet, proporcionar economia de tráfego de dados e consequente redução de custo com a Internet [3].

O protocolo Wi-Fi Direct (WD) é uma das tecnologias mais promissoras para proporcionar essa conveniência por não demandar recursos de hardware além dos utilizados por uma comunicação Wi-Fi. Criado e mantido pela Wi-Fi Alliance [7] o WD é uma extensão do protocolo Wi-Fi que permite a conexão direta entre dispositivos sem a necessidade de um Access Point (AP). Incorporado ao sistema operacional Android a partir da versão 4, o WD é 
uma opção para a conexão direta entre dispositivos. Diferente do Bluetooth e Near Field Communication (NFC), o WD não precisa de hardware específico para seu funcionamento, sendo apenas necessário os recursos para conexão Wi-Fi, que são praticamente um padrão nos dispositivos móveis, o que viabiliza sua utilização mesmo em dispositivos de baixo custo como os projetados para compor redes de sensores ou para atender demandas de comunidades carentes.

Entretanto, o WD é pouco adotado por usuários ou aplicativos. Um dos motivos é que o estabelecimento de uma conexão, que é um processo mais complexo que o uso da Internet. Uma vez que os usuários precisam se envolver diretamente no processo de conexão para a seleção e autorização de dispositivos. O conhecimento para estabelecer a conexão e o tempo gasto inviabilizam o uso cotidiano e limitam os cenários de uso do WD. Para lidar com essas limitações, este trabalho introduz o conceito de Spontaneous Connection (SC), que reduz a necessidade de conhecimento sobre conexão direta entre dispositivos, reduzindo a quantidade de passos para estabelecer a comunicação. Como contribuições este artigo apresenta uma arquitetura para SC entre dispositivos e a implementação do módulo de conexão e transferência de dados como uma extensão do WD, chamada de WDSC - Wi-Fi Direct with Spontaneous Connection. Por fim, a implementação de um aplicativo que demonstra como aplicações podem se beneficiar do WDSC.

O trabalho está organizado da seguinte forma. Na seção 4.1 é apresentada uma visão geral do funcionamento do WD no Android. Na seção 4.2 são apresentados os trabalhos relacionados e em seguida, na seção 4.3, é introduzido o conceito de SC. Na seção 4.4, as limitações encontradas e implementação WDSC. Logo é realizada a validação na seção 4.5 e, após isso, a discussão e conclusão do trabalho na seção 4.6, com um resumo e esboço do planejado para futuras evoluções.

\subsection{Visão Geral do Wi-Fi Direct no Android}

Para que uma aplicação consiga utilizar o WD no Android, ela deve criar uma instância da classe WifiP2pManager, que é responsável por estabelecer um canal de comunicação entre a aplicação e o serviço do WD. Operações como descoberta de dispositivos, solicitação de abertura ou fechamento de conexão, entre dispositivos, são acionados pelas aplicações através da sua instância local da classe WifiP2pManager, que envia esses comandos ao serviço do WD, implementado pela classe WifiP2pServiceImpl. 
O serviço do WD funciona de maneira semelhante ao Wi-Fi ou seja, quando o Wi-Fi estiver ativo no dispositivo, qualquer aplicação que possua as devidas permissões pode acessálo, sendo executado por apenas uma instância. Essa instância, única, recebe as solicitações de todas as aplicações que desejam utilizar do WD, para estabelecer uma rede entre dispositivos próximos. Ao estabelecer uma conexão com um dispositivo, o serviço do WD compartilha os dados da conexão realizada com todas as aplicações que estiverem lhe consumindo através da instância da classe WifiP2pManager.

A demonstração do processo de estabelecimento de uma conexão entre dois dispositivos é apresentada a seguir. A Fig. 2 mostra o dispositivo A solicitando conexão com o dispositivo B. Por sua vez, a Fig. 3 mostra que o dispositivo B exibe uma caixa de diálogo ao usuário para que seja autorizada a conexão manualmente.

Figura 2 - Solicitação de conexão do dispositivo A ao B

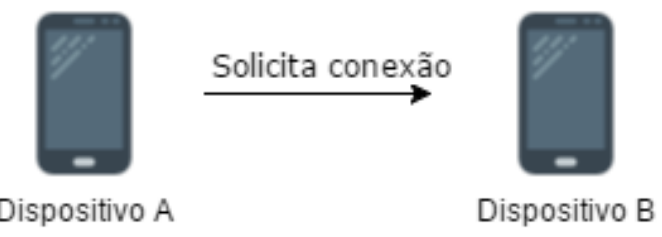

Fonte: Ricardo César Corrêa, 2016

Figura 3 - Dispositivo B exibe notificação de solicitação de conexão do dispositivo A ao usuário, que deve aceitar manualmente a conexão

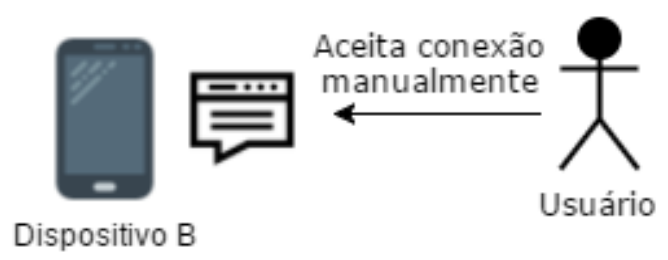

Fonte: Ricardo César Corrêa, 2016

Ao aceitar a solicitação de conexão, o dispositivo B envia a confirmação ao dispositivo A, conforme Fig. 4. Quando o dispositivo recebe a confirmação e estabelece a comunicação, ele passa a realizar um broadcast com os dados para todas as aplicações em execução, na Fig. 5. A partir dessas informações de conexão, as aplicações podem então criar um socket para iniciar a transferência de dados entre os dispositivos. 
Figura 4 - Dispositivo B envia confirmação do aceite da conexão ao dispositivo A

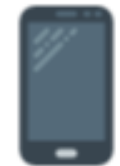

Dispositivo A

\section{Envia confirmação}

$\longleftarrow$

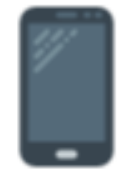

Dispositivo B

Fonte: Ricardo César Corrêa, 2016

Figura 5 - Dispositivo A realiza um broadcast com as informações da conexão para todas as aplicações em execução

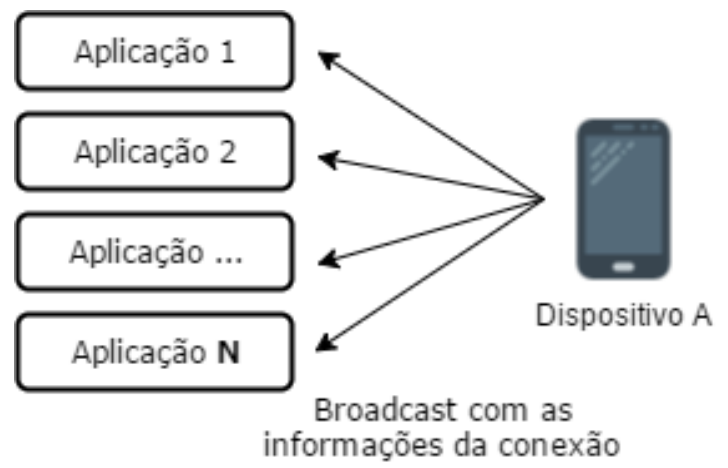

Fonte: Ricardo César Corrêa, 2016

O diagrama dos módulos envolvidos em uma conexão pelo WD é demonstrado na Fig. 6. O processo é iniciado no dispositivo A por uma de suas aplicações, ao realizar uma chamada a operação connect da instância local da classe WifiP2pManager. A operação connect envia uma solicitação de conexão para a classe local WifiP2pServiceImpl, serviço do WD, que envia essa solicitação ao dispositivo alvo. A classe WifiP2pServiceImpl, no dispositivo alvo, recebe a solicitação de conexão e exibe uma caixa de diálogo, notificando o usuário sobre a necessidade de autorizar a conexão. As telas de solicitação de conexão e a exibição dessa notificação, da forma como aparecem no Android são apresentadas na Fig. 7.

A implementação atual da Application Programming Interface (API) do WD não prevê conexão direta sem a intervenção humana. Dessa forma, a alternativa de comunicação sem a necessidade de acesso à Internet proporcionada pelo WD fica limitada a conexões individuais e à demanda aprovação a priori. Esse tipo de autorização cria a percepção de segurança de uma conexão autorizada, mas inibem potenciais utilizações e a simplificação do processo de comunicação entre dispositivos. 
Figura 6 - Funcionamento interno do WD

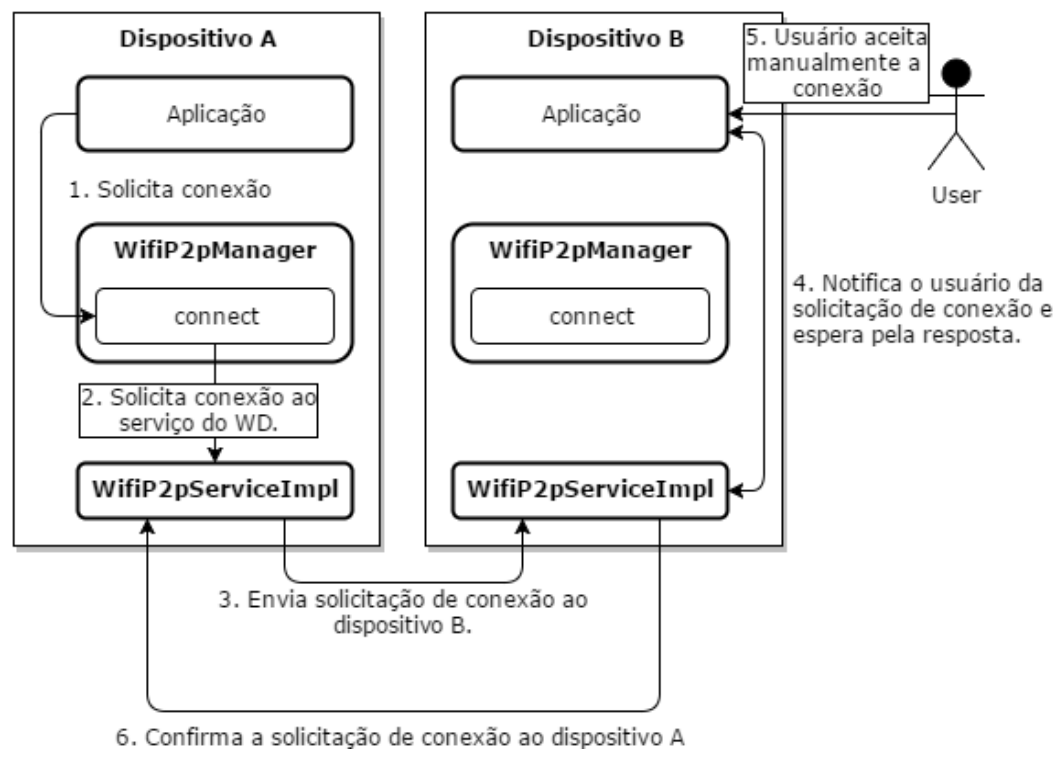

Fonte: Ricardo César Corrêa, 2016

Figura 7 - Solicitação de conexão pelo dispositivo A (a) ao dispositivo B (b)

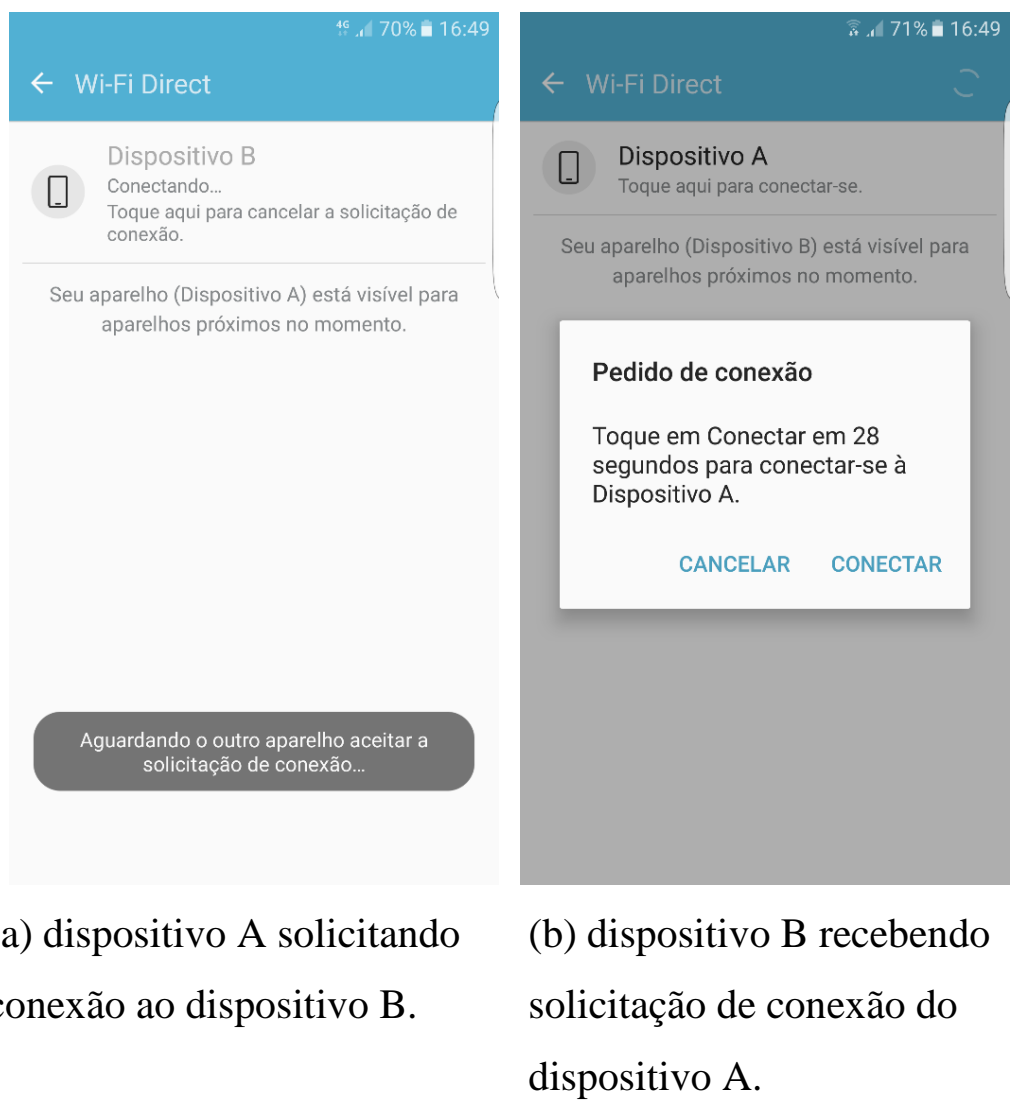

Fonte: Ricardo César Corrêa, 2016 
Como forma de ampliar as possibilidades de uso da comunicação direta entre dispositivos móveis, este artigo propõe uma extensão ao protocolo, chamado de Wi-Fi Direct with Spontaneous Connection (WDSC). Com ele, a conexão entre dispositivos pode ocorrer de forma automática sempre que dispositivos estejam próximos. Além de preservar a capacidade do usuário de decidir se deseja utilizar ou não os dados trocados por essas conexões. Ainda como contribuição, este trabalho também apresenta o desenvolvimento de um aplicativo, chamado de Together, que ilustra como o WDSC pode ser utilizado na prática para prover comunicação simplificada e instantânea.

\subsection{Trabalhos Relacionados}

A conexão no protocolo WD é composta por passos de validação como o mecanismo, chamado de Wi-Fi Protected Setup [36], que demanda um Service Set Identifier (SSID) e senha para a autorização da conexão. No Android esse processo é feito com a troca de tokens de acesso ou pelo pressionar de botões de confirmação nos dispositivos envolvidos para confirmar a conexão [9].

$\mathrm{Na}$ literatura, alguns trabalhos propuseram alternativas para simplificar a comunicação. No trabalho [17], é criado um Agente Gerenciador de Conexão. Por meio dele os dispositivos trocam e armazenam informações de conexões e dispositivos autorizados em um banco de dados local para simplificar futuras conexões entre dispositivos conhecidos. Já o artigo [13] oferece uma alternativa de criptografia para permitir a conexão entre dispositivos. Em sua proposta, a informação do SSID e preshared key, necessários para conexão, são criptografados e embutidos nas mensagens do network service discorvery broadcast do serviço de descoberta. Assim todos os dispositivos dentro da área de cobertura recebem tais informações e armazenam localmente para utilizá-la posteriormente para a conexão. O trabalho [17] propõe uma maneira para aceitar conexões por meio do acesso a funções privadas e ocultas da API do WD usando reflexão Java. Abordagem que permite acesso e modificação do comportamento de aplicações em execução em uma máquina virtual Java. Entretanto, esse recurso não é oficialmente suportado pelo Android e pode não funcionar em futuras versões.

Os artigos [13] e [17] modificam a mensagem do network service discovery broadcast para enviar mais informações do que definido por padrão. Entretanto a modificação da mensagem do serviço de descoberta ou o acesso a funções privadas e ocultas do sistema operacional pode causar a quebra da funcionalidade em versões futuras e pode ser considerada 
invasivo, já que modifica um comportamento da API do WD sem o consentimento do usuário, sendo um pré-requisito para as aplicações desenvolvidas para Android informarem e solicitarem as permissões adequadas para o seu funcionamento.

Para proporcionar a conexão sem exigir autorização prévia e garantir que apenas aplicações com permissões pré-definidas possam usufruir desse novo recurso, propomos o conceito de conexão espontânea, apresentado a seguir.

\subsection{Conexão Espontânea}

Para lidar com as questões de segurança, protocolos como o Bluetooth e WD demandam a autorização manual do usuário para evitar conexões indesejadas e com dispositivos desconhecidos. A necessidade desse tipo de intervenção manual do usuário vem do conceito de conexão que visa garantir maior segurança na conexão entre os dispositivos. A Fig. 8 mostra o processo de autorização de conexão implementados pelos protocolos Bluetooth e WD. Nele, a solicitação de conexão pode ser realizada por uma aplicação ou usuário, porém não pode ser aceita automaticamente no dispositivo alvo sem a intervenção manual. Essa abordagem visa reduzir a vulnerabilidade dos dispositivos, pois uma vez estabelecida a conexão as aplicações podem ter acesso aos dados do dispositivo. Entretanto, após a autorização, mesmo que equivocada, a conexão pode ser utilizada por qualquer aplicativo dentro do Android.

Figura 8 - Conceito de conexão abordado atualmente

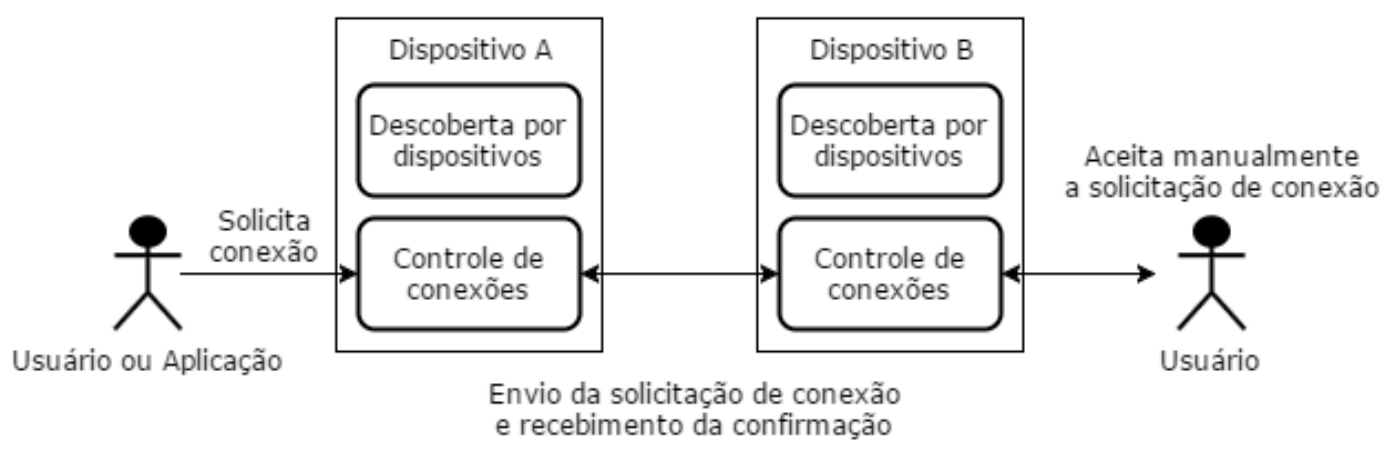

Fonte: Ricardo César Corrêa, 2016

A inclusão da fase de autorização da conexão restringe a comunicação entre dispositivos em cenários de conexões entre dois dispositivos. A inclusão de mais dispositivos exigiria a autenticação dos vários dispositivos envolvidos. O que tornaria o processo burocrático e 
demorado. Para ampliar as possibilidades de comunicação entre dispositivos próximos, esse trabalho propõe o conceito de Conexão Espontânea, também referida neste trabalho por Spontaneous Connection - SC, tradução livre para a língua inglesa.

Para que a conexão espontânea aconteça, basta que os dispositivos possam ser identificados. Dessa forma, a SC permite que todos os dispositivos próximos sejam potenciais partes de uma rede de comunicação, sem que exista a exigência de uma autorização antes do estabelecimento da conexão. Assim, a comunicação pode acontecer de forma direta.

Com a SC, um dispositivo pode transmitir dados para mais de um dispositivo e aumentar as possibilidades de aplicação para a comunicação entre dispositivos. Uma vez que os dispositivos estejam próximos, a conexão pode ser estabelecida e a troca de dados pode ser iniciada.

Para lidar com questões de segurança, a SC propõe uma abordagem alternativa para a realização de conexão com o uso de um Controle de Acesso em conjunto com um Contêiner, para o armazenamento de dados. O Controle de Acesso permitirá que aplicações ou dispositivos com as devidas permissões possam se conectar automaticamente entre si e trocar dados. Por sua vez, os dados bloqueados para acesso ou execução, criando um Contêiner para a retenção do dados. Os dados no Contêiner somente poderão ser acessados com as autorizações configuradas no Controle de Acesso ou mesmo pelo usuário, apresentado um esboço na Fig. 9.

Figura 9 - Conceito de conexão e transferência de dados proposto

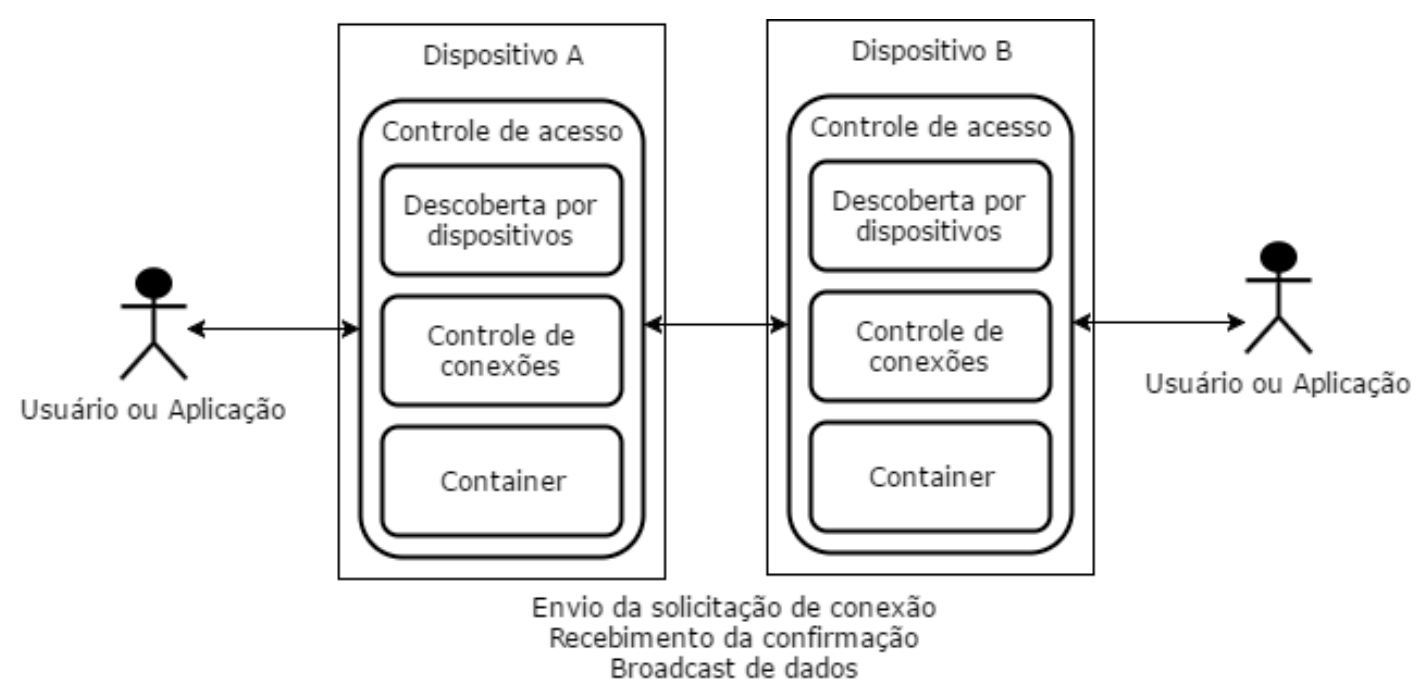

Fonte: Ricardo César Corrêa, 2016 
Assim, as conexões e as trocas de dados entre os dispositivos podem acontecer de forma contínua e os dados recebidos só poderão ser utilizados de acordo com autorizações previamente definidas. Com a SC, o usuário poderá decidir o nível de autorização desejada para os dados recebidos, baseando-se em 7 tipos de autorizações ou combinações delas, da mais livre e automática até a mais específica e restritiva. As autorizações criadas pelo SC são:

1. Autorização automática: modo de autorização é o menos restritivo, não haverá verificação de permissão para realização da conexão e nem para os dados transferidos entre os dispositivos. Esse modo de autorização é importante para ambientes seguros. Ou seja, não existe preocupação com acessos indevidos. Nesse caso a conexão acontece de forma simples e sem interferência manual.

2. Autorização geo-referenciamento: modo de autorização permite criar regiões de confiança para conexões e uso de dados.

3. Autorização por tipo de dispositivo: autorização que viabiliza a comunicação entre IoT's, como: Impressoras, sensores, eletrodomésticos, entre outros.

4. Autorização por dispositivo: essa opção identifica o dispositivo como seguro. $\mathrm{O}$ que autoriza automaticamente a conexão e transferência de dados oriunda do dispositivo.

5. Autorização por aplicação: autorização que permite que aplicações específicas realizem a conexão automática e troquem dados entre si sem que o arquivo seja retido no Container para aguardar autorização de uso. Assim, aplicativos como redes sociais, mensagens instantâneas entre outros manterão fluxo contínuo de comunicação entre os dispositivos.

6. Autorização por tipo de arquivo: permite que determinados tipos de arquivos não necessitem de autorização para a liberação de seu uso. Assim, o usuário pode definir que arquivos como mensagens, músicas ou propagandas lhe sejam enviadas sem a necessidade de confirmação prévia.

7. Autorização por arquivo: com esse modo de autorização, os arquivos armazenados no Container só poderão ser utilizados se houver autorização, por arquivo. Nesse caso, uma janela de confirmação mostrará detalhes do arquivo, como: nome, data de recebimento, dispositivo de origem, local de recebimento e tipo de arquivo. Caso autorizado, o arquivo é liberado para uso por qualquer aplicação que o solicitar. Caso o uso seja negado, o arquivo é removido do dispositivo. 
As autorizações podem ser combinadas para aumentar a segurança e comodidade do usuário. Como exemplo, a combinação das autorizações 2, 3 e 6 pode autorizar o uso automático de mensagens oriundas de impressoras que se encontram em um escritório.

\subsection{Wi-Fi Direct with Spontaneous Connection}

Este trabalho avaliou a viabilidade de implementar uma extensão do protocolo WD para inclusão do conceito de SC e, a partir disso, criar o WDSC - Wi-Fi Direct with Spontaneous Connection. Para tanto foram implementados os processos de conexão automática e de armazenamento e retenção dos dados transmitidos no Contêiner.

O principal objetivo do WDSC é o estabelecimento de conexões e transferência de dados entre dispositivos sem que seja necessária uma confirmação humana. E dessa forma expandir os cenários de aplicação de comunicação entre dispositivos. Para tanto, esta seção apresenta os requisitos e implementação necessários para que a comunicação seja estabelecida. Inicialmente, são apresentadas as limitações da implementação atual do protocolo WD no Android e em seguida a solução proposta.

\section{A. Limitações do WD}

O WD possui um serviço responsável por gerenciar as conexões solicitadas pelas aplicações, internas ou externas, chamado de Serviço do WD. As solicitações são realizadas pelas aplicações internas através da criação de uma instância da classe WiFiP2pManager. Assim, cada aplicação possui sua própria instância da classe WifiP2pManager. Essa classe envia comandos ao Serviço do WD que, de acordo com a solicitação, realiza operações como, descoberta de dispositivos, solicitação para conectar ou desconectar com outro dispositivo.

Quando o Serviço do WD possui informação para transmitir, como dados de uma conexão ou de dispositivos descobertos, ele envia uma mensagem de broadcast com tais informações para todas as aplicações em execução. Esse comportamento permite que, mesmo se uma aplicação solicitar uma conexão e obter a confirmação do dispositivo alvo, outras aplicações receberão os dados da conexão de qualquer forma, inclusive as que não possuem autorização. Esse comportamento da atual implementação do WD faz com que não seja possível realizar o isolamento por aplicação de uma conexão, ou seja, uma vez estabelecida a conexão todas as aplicações receberão os dados enviados em forma de broadcast.

Todas as aplicações desenvolvidas para Android possuem um arquivo chamado de manifesto, nele são descritas as permissões necessárias para a aplicação, como permissão para 
acessar o cartão de memória ou possuir acesso ao Wi-Fi. Para que uma aplicação no Android, de acordo com o que está configurado em seu manifesto, altere um comportamento interno do WD, como, automatizar a conexão, demanda a criação de um isolamento por aplicação. Uma vez que uma aplicação A pode ter a autorização do usuário para um comportamento específico e a aplicação B não. Essa declaração de confiança do usuário em uma determinada aplicação é definida no Android como uma permissão solicitada ao usuário para acessar recursos do dispositivo. Além disso, em tempo de execução, a aplicação deve solicitar a autorização manual ao usuário para acesso a cada um dos recursos descritos no manifesto.

Outro limite do protocolo é que o WD apenas atribui IP aos dispositivos, não estão disponíveis recursos para transferência de dados, serviço, que deve ser realizado pelo desenvolvedor. Ao receber a informação de IP do Serviço do WD, o desenvolvedor necessita criar um Socket com essa informação para então realizar a transferência de dados entre os dispositivos. Essa abordagem delega o gerenciamento dos dados trafegados entre os dispositivos para cada aplicação. Assim, para que as informações transferidas sejam interpretadas é necessário que cada dispositivo possua instalada a aplicação correspondente. Uma vez que não há mecanismo genérico para isso.

\section{B. Arquitetura do WDSC}

Para implementar o conceito de SC no protocolo WD foi implementado o isolamento de conexão por aplicação e mecanismo para o broadcast dos dados que desejam ser trocados entre os dispositivos, simulando uma conexão automática e transferência de dados entre os dispositivos conectados. Para isso, a seguir é detalhada a forma atual de funcionamento do WD e, em seguida, os pontos alterados para permitir esses comportamentos.

Conexão automática: A forma do WD de gerenciar conexões é detalhada na Fig. 10. No momento de inicialização da aplicação é criada uma instância da classe WifiP2pManager e então chamada a sua operação initialize, responsável por criar um canal de comunicação com a classe WifiP2pServiceImpl, Serviço do WD. Em seguida, é chamada a operação discoverPeers, responsável por descobrir dispositivos próximos. A operação discoverPeers envia a mensagem DISCOVER_PEERS ao Serviço do WD, que está no estado de P2pEnabledState. Esse estado, ao receber a essa mensagem, envia uma mensagem de broadcast para todos os dispositivos próximos. Ao receber uma resposta dos dispositivos próximos, o Serviço do WD aciona a operação sendPeersChangedBroadcast, da qual envia uma mensagem de broadcast, WIFI_P2P_PEERS_CHANGED_ACTION, para todas as aplicações em execução, com os dados dos dispositivos encontrados. 
Figura 10 - Fluxo atual, simplificado, da solicitação de dispositivos próximos e conexão

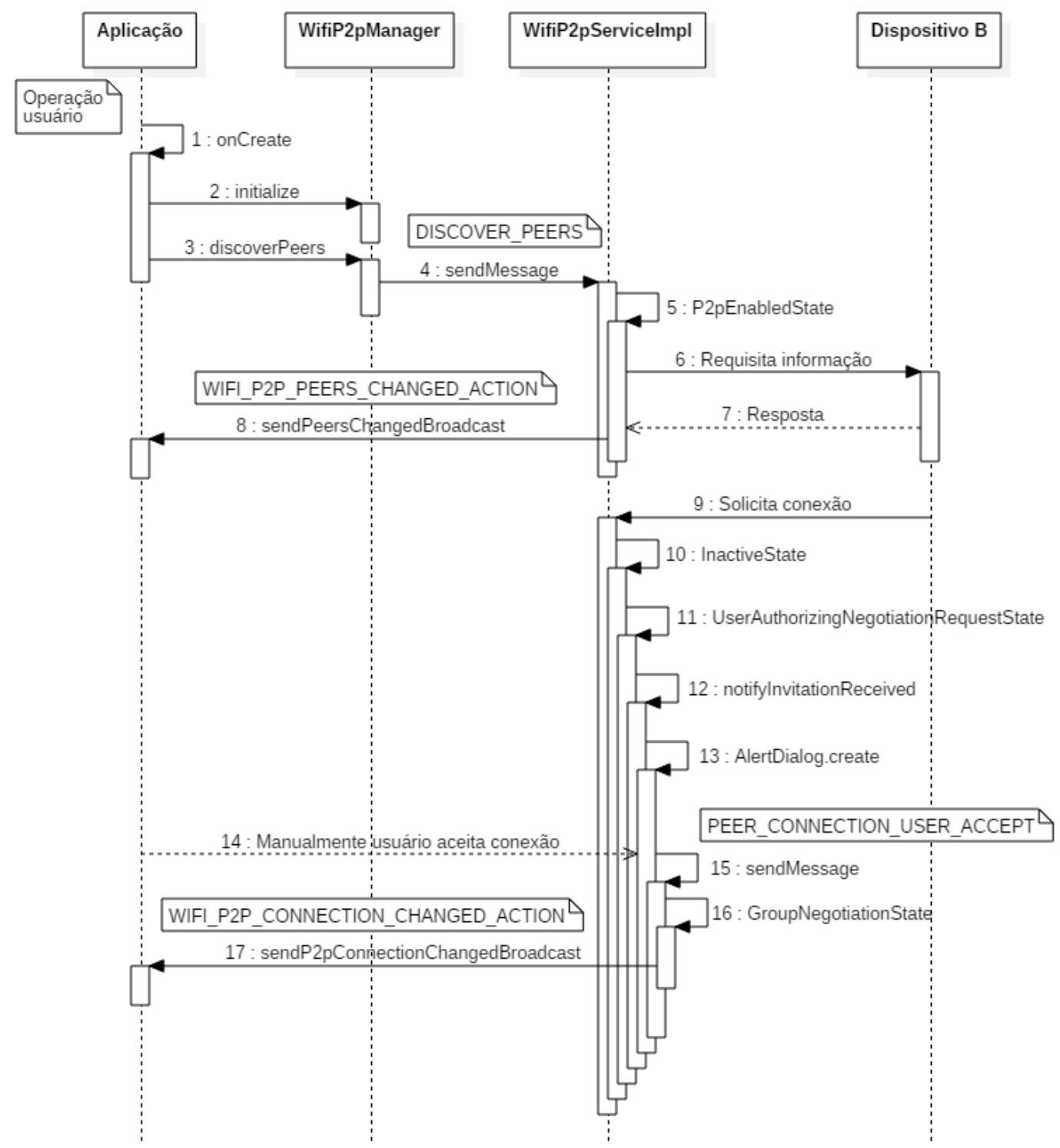

Fonte: Ricardo César Corrêa, 2016

Da mesma forma, essas operações são executadas no Dispositivo B, que com a informação dos dispositivos existentes, solicita uma requisição de conexão ao dispositivo alvo, que está no estado InactiveState. Ao receber a solicitação de conexão, o atual estado do serviço do WD é alterado para o estado de UserAuthorizingNegotiationRequestState, que, ao entrar nesse estado, aciona a operação notifyInvitationReceived, responsável por exibir uma caixa de diálogo da conexão solicitada ao usuário. Exibida a caixa de diálogo, o usuário deve manualmente indicar se aceita a requisição de conexão do Dispositivo B. 
Ao aceitar a conexão, é enviada a mensagem PEER_CONNECTION_USER_ACCEPT, que altera para o estado de GroupNegotiationState. Esse estado aciona a operação sendP2pConnectionChangedBroadcast que envia para todas as aplicações em execução do dispositivo, em forma de broadcast, a mensagem WIFI_P2P_CONNECTION_CHANGED_ACTION, com os dados da conexão embutidos nessa mensagem.

Para tornar possível a conexão espontânea, a operação initialize da classe WifiP2pManager e as operações sendPeersChangedBroadcast, sendP2pConnectionChangedBroadcast e notifyInvitationReceived da classe WifiP2pServiceImpl foram modificadas com a inclusão de uma nova permissão ao sistema operacional Android, chamada de CHANGE_WIFI_P2P_CONNECTION. Essa permissão deve ser adicionada ao manifesto das aplicações que desejarem utilizar o comportamento de conexão automática do WD.

Na Fig. 11 é destacado em azul os pontos alterados no fluxo de solicitação de dispositivos próximos e conexão. Ao realizar a chamada para a operação initialize da classe WifiP2pManager é registrada a intenção WIIF_P2P_CONNECTION_REQUEST_CHANGE_ACTION, usada posteriormente no momento da conexão. Em seguida a aplicação que criou a instância da WifiP2pManager é registrada no serviço, através da operação registerApplication. O serviço armazena uma lista das aplicações registradas, com um booleano para dizer se a aplicação possui permissão para conexão automática e uma lista com os endereços dos dispositivos conectados nessa aplicação.

Ao receber uma solicitação de conexão, a operação notifyInvitationReceived é acionada no Serviço do WD, que executa a operação sendP2pConnectionRequestChangeBroadcast. Ela por sua vez envia um broadcast da mensagem WIFI_P2P_CONNECTION_REQUEST_CHANGE_ACTION. Todas as aplicações registradas para receber o broadcast dessa intenção, anteriormente registradas na operação initialize da classe WifiP2pManager, irão verificar se o aplicativo em execução possui permissão para conexão automática. Caso possuam a autorização, será executada a operação setApplicationPermission do Serviço do WD. Essa operação é a responsável por atualizar a configuração da aplicação e informar ao serviço sobre a autorização para conexão espontânea. Após essa etapa, o Serviço do WD, com o uso da operação notifyInvitationReceived verifica se existe alguma aplicação com permissão para conexão espontânea. Se existir autorização é 
disparada a mensagem PEER_CONNECTION_USER_ACCEPT. Assim, a conexão é confirmada sem a necessidade de intervenção do usuário.

Figura 11 - Fluxo proposto para solicitação de dispositivos próximos e conexão

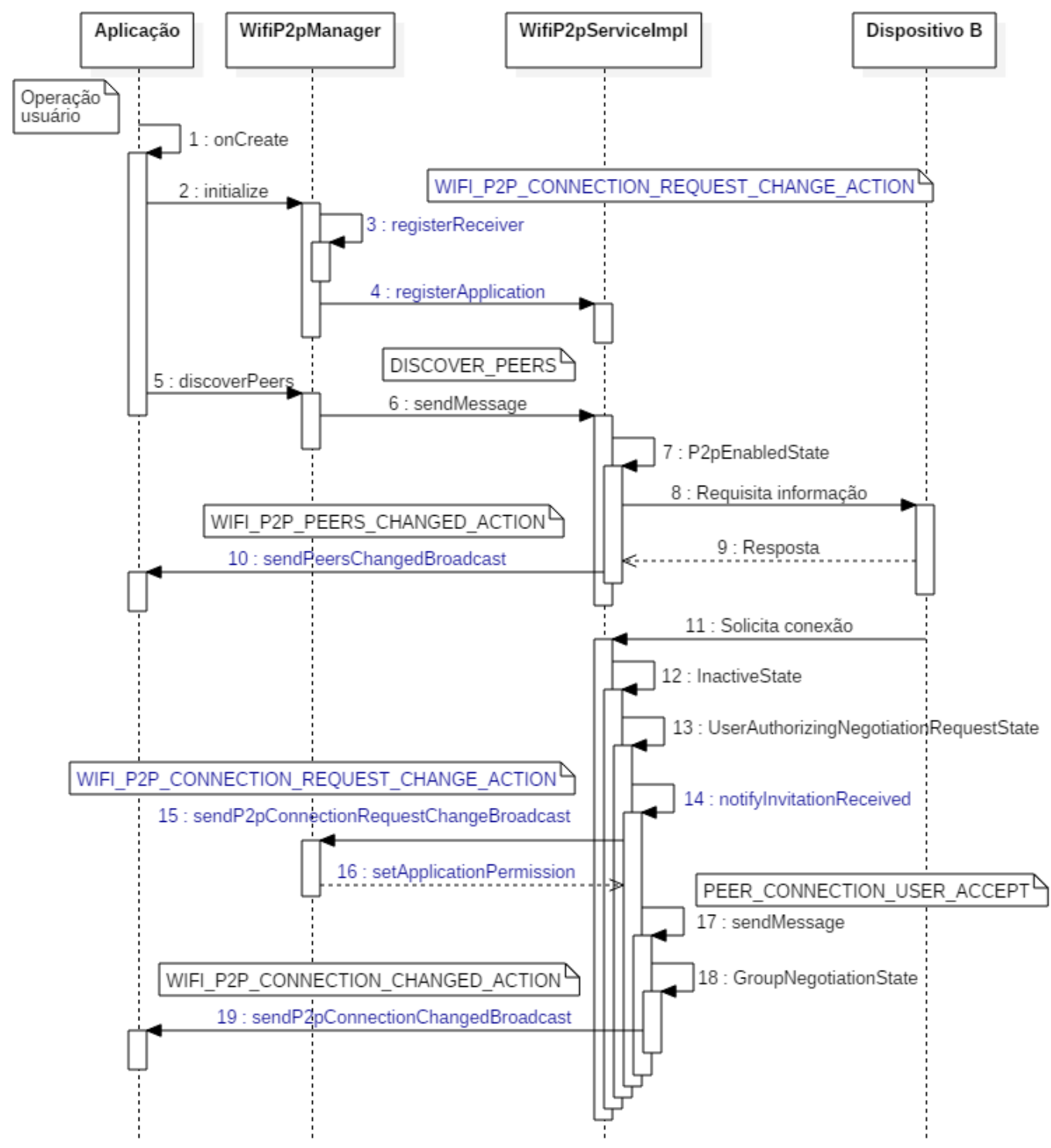

Fonte: Ricardo César Corrêa, 2016

Porém se for identificada alguma aplicação que não possua as permissões necessárias, uma caixa de diálogo é exibida para confirmação manual da conexão, mesmo após a conexão já ter sido aceita. Com a confirmação do usuário, todas as aplicações que não tiverem a permissão para conexão automática terão a sua lista de dispositivos atualizada com o endereço do dispositivo que podem receber os dados de conexão. 
A partir disso, as operações sendPeersChangedBroadcast e sendP2pConnectionChangedBroadcast indicam quais informações podem ser enviadas para cada aplicação. Com a lista de aplicações registradas é possível direcionar o broadcast de dados para uma determinada aplicação e, a partir das configurações dessa aplicação, decidir quais dados a aplicação pode utilizar.

Com essa implementação, o conceito do controle de conexões do SC é oferecido, com o controle de que a conexão automática só estará disponível para aplicações autorizadas. Além disso, no caso de aplicações sem a permissão para conexão automática, o processo de autorização com o uso da confirmação manual ocorrerá normalmente. Com o isolamento dos dados da conexão, por aplicação, é possível que aplicações com ou sem a permissão coexistam dentro da atual implementação do serviço do WD no Android.

\section{B.2. Contêiner}

Para a implementação do conceito de Contêiner, o Serviço do WD foi adaptado para que, no momento da criação de uma conexão, seja também criado um Socket com a informação de IP do dispositivo. Dessa forma é estabelecido um canal de comunicação com o dispositivo alvo para permitir a transferência de dados. Assim, os dados transmitidos são armazenados de forma bloqueada, no Contêiner, de cada dispositivo. Uma vez no Contêiner, os dados somente podem ser acessados pelas aplicações que tiverem a permissão P2P_READ_DATA, criada para este fim.

O Contêiner foi criado como um serviço do sistema operacional. Nele, são registrados e armazenados os dados recebidos ou que devem ser transmitidos para outros dispositivos. Para que uma aplicação localmente registre dados para enviar para outros dispositivos, ela deve ter a permissão P2P_WRITE_DATA. Permissão que autoriza a aplicação quanto ao registro dos dados a serem enviados para outros dispositivos. O Serviço do WD recuperará no Contêiner os dados a serem enviados para o dispositivo alvo. Da mesma forma, no dispositivo alvo, o Serviço do WD irá receber os dados enviados e os armazenarão no Contêiner local.

Essa implementação do WDSC demonstra a viabilidade do uso do conceito de SC como extensão do protocolo WD. Para isso, implementamos o conceito parcialmente, permitindo a conexão automática e broadcast de dados entre os dispositivos. 


\subsection{Aplicativo com o uso de conexão espontânea - Together}

Como forma de demonstrar os benefícios de uso do WDSC, tanto pelos desenvolvedores, quanto pelas aplicações, foi implementada uma aplicação para Android, chamada de Together. Ela estabelece a conexão espontânea com o uso do WDSC e faz troca de dados entre os aplicativos. $\mathrm{O}$ aplicativo Together possui em seu manifesto as permissões criadas para o WDSC. Sendo elas: CHANGE_WIFI_P2P_CONNECTION, P2P_READ_DATA e P2P_WRITE_DATA, que permitem que a conexão automática seja realizada, com leitura e registro dos dados no Contêiner, respectivamente.

De forma transparente ao usuário, o Together realiza uma varredura para detectar dispositivos próximos. Com a lista de dispositivos próximos, o Together estabelece a conexão com cada um dos dispositivos encontrados. Na Fig. 12 é apresentada a aplicação com a conexão realizada de forma automática entre dois dispositivos.

Figura 12 - Aplicação Together com a conexão entre os dispositivos A e B realizada de forma espontânea

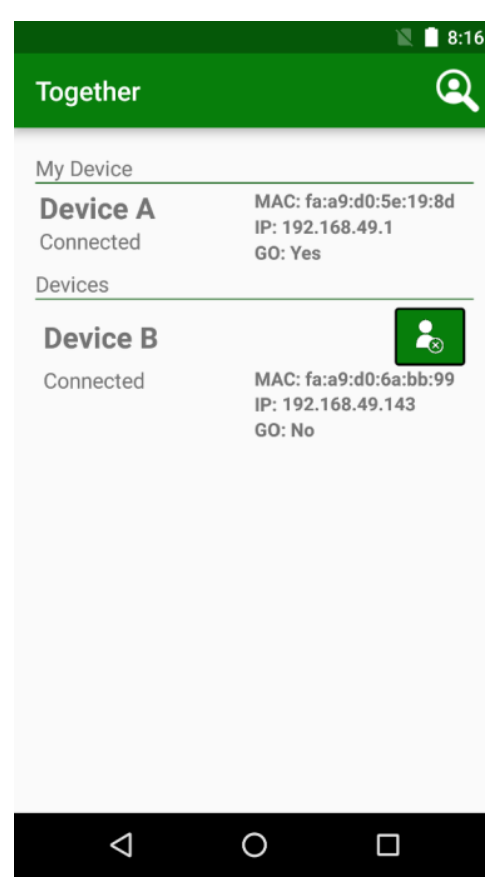

(a) Dispositivo A
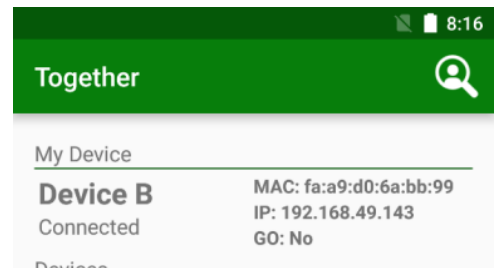
Devices

\begin{tabular}{|c|c|}
\hline Device A & $i+i$ \\
\hline Connected & $\begin{array}{l}\text { MAC: fa:a9:d0:5e:19:8c } \\
\text { IP: } 192.168 .49 .1 \\
\text { GO: Yes }\end{array}$ \\
\hline
\end{tabular}

$\triangleleft \quad \bigcirc \quad \square$

(b) Dispositivo B. 
Com a conexão estabelecida, o Serviço do WD está pronto para enviar dados e começar a receber dados que estão sendo transmitidos. Nesse caso, os dados são gerenciados pelo Contêiner. A Fig. 13, mostra os dados armazenados no Contêiner e percentual de transferência. De posse da permissão P2P_READ_DATA, o Together pode realizar a leitura desses.

Figura 13 - Lista de arquivos do Contêiner com a porcentagem de dados transferidos de arquivos oriundos de outros dispositivos

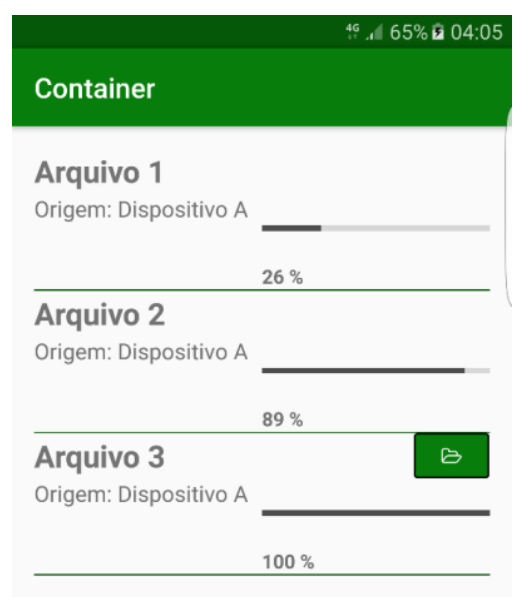

Fonte: Ricardo César Corrêa, 2016

\subsection{Considerações finais}

A comunicação direta entre dispositivos próximos é uma funcionalidade que apoia diferentes cenários para o uso dos dispositivos mesmo em situação de ausência de conexão. Entretanto, protocolos de comunicação clássicos, como Bluetooth e WD, exigem que cada conexão seja autorizada pelo usuário, antes que seja estabelecida. Este trabalho apresentou o conceito de SC, que permite a conexão entre dispositivos e troca de dados de forma automática. Essa abordagem faz uso de um Contêiner para gerenciar os dados recebidos durante uma conexão. Dessa forma, o dispositivo pode receber dados sem autorização prévia. Entretanto, os dados somente serão utilizados se a aplicação possuir autorização de leitura dos dados. Assim, a comunicação pode ser realizada de maneira automática, sem retirar do usuário a decisão se 
deseja ou não utilizar os dados. Para implementação do conceito proposto foi apresentada uma extensão do protocolo WD para Android, chamada de WDSC. Com essa extensão, os aplicativos podem se comunicar de forma direta e simplificada. Também foi apresentada o aplicativo Together que demonstra como um aplicativo pode utilizar os recursos da comunicação espontânea disponíveis por essa extensão.

A experiência de criar o WDSC mostrou a necessidade de atender requisitos do protocolo WD que não são necessários em uma abordagem pura do conceito de conexão espontânea. Portanto, como trabalhos futuros será implementado um novo protocolo de comunicação, alternativo ao WD, para que os conceitos da conexão espontânea possam ser explorados de forma direta. 


\section{Conclusões Finais}

Neste trabalho foi proposto o conceito de Spontaneous Connection (SC), o qual tem por objetivo tornar mais prática a conexão e transferência de dados entre dispositivos. Para tanto, uma extensão do protocolo WD foi proposta e implementada, chamada de WDSC. O WDSC possibilita que aplicações para dispositivos móveis possam usufruir dos recursos da conexões automáticas e transferência de dados entre dispositivos móveis próximos através da utilização da tecnologia WD, permitindo ampliar as possibilidades de uso dos dispositivos móveis mesmo em áreas sem Internet.

A experiência de evolução do código do sistema operacional Android experimentou uma série de desafios. Entre eles, se destacam a falta de documentação do código e o grande tempo necessário para compilar e executar testes. Cada compilação em um notebook com processador Intel i7, 16GB de memória ram e disco rígido de solid state drive (SSD) leva aproximadamente 2 horas para término do processamento

A escolha de extensão do protocolo WD expôs o projeto às mudanças ocorridas na atualização da versão 5 para a versão 6 ao decorrer do projeto, o que exigiu aprendizado da nova estrutura e atualizações na implementação da WDSC, da qual, parte utilizada pela implementação proposta, se tornou obsoleta com a atualização de versão. Ainda em relação ao WD, sua arquitetura restringe a realização de rotinas que modifiquem o seu comportamento por aplicação. Mesmo assim, o isolamento foi realizado de forma suficiente para mostrar a viabilidade e vantagens da SC.

Como contribuições, este trabalho produziu um artigo de revisão sistemática que apresenta limitações e aplicações do protocolo WD, o que pode ser utilizado para definição de pesquisas que visam tanto evoluir o protocolo quanto criar novas abordagens de comunicação. Também foi proposto o conceito de conexão espontânea que amplia as possibilidades de comunicação entre dispositivos, o que pode ter impacto tanto na redução dos custos de troca de dados quanto apoiar o surgimento de novas aplicações. A implementação da conexão espontânea como extensão do protocolo WD contribui como prova de conceito dessa alternativa de conexão. Por fim, o aplicativo WD apresenta como contribuição a demonstração de como aplicativos também podem fazer uso da conexão espontânea para oferta de funcionalidade.

Para trabalho futuro, estamos trabalhando na implementação de um novo protocolo de comunicação baseado nos conceitos de conexão espontânea, o que permitirá explorar 
características como o isolamento por aplicação, para garantir exclusividade de uso dos recursos pelas aplicações do dispositivo autorizados. 


\section{Referências}

1 CISCO Visual Networking Index: Global Mobile Data Traffic Forecast Update, 20152020. Cisco, 2016. Disponível em:

<http://www.cisco.com/c/en/us/solutions/collateral/service-provider/visual-networkingindex-vni/mobile-white-paper-c11-520862.pdf>. Acesso em: 05 Fev. 2016.

2 SANKARAN, C. B. Data Offloading Techniques in 3GPP Rel-10 Networks: a Tutorial.

IEEE Communications Magazine, v. 50, p. 46-53, Jun. 2012. Disponível em:

$<$ http://ieeexplore.ieee.org/xpl/articleDetails.jsp?arnumber=6211485>. Acesso em: 10 Out. 2015.

3 PYATTAEV, A. et al. Network-Assisted D2D Communications: Implementing a Technology Prototype for Cellular Traffic Offloading. 2014 IEEE Wireless Communications and Networking Conference (WCNC). Anais... . p. 3266-3271, 06-09, Apr. 2014. Istanbul: IEEE. Disponível em:

<http://ieeexplore.ieee.org/xpl/articleDetails.jsp?arnumber=6953070>. Acesso em: 10 Out. 2015.

4 KITCHENHAM, B; Guidelines for Performing Systematic Literature Reviews in Software Engineering, Technical Report EBSE-2007-01, Keele University, United Kingdom, 2007.

5 ZAMBONI, A. et al. Start: Uma ferramenta computacional de apoio à revisão sistemática. Congresso Brasileiro de Software (CBSoft'10). Set. 2010.

6 CISCO Visual Networking Index: Global Mobile Data Traffic Forecast Update, 20142019. Cisco, 2015. Disponível em:

<http://www.cisco.com/c/en/us/solutions/collateral/service-provider/ip-ngn-ip-nextgeneration-network/white_paper_c11-481360.pdf>. Acesso em: 20 Dez. 2015.

7 Wi-Fi Alliance. Disponível em: <http://www.wi-fi.org/>. Acesso em: 21 Jan. 2016.

8 SHANIN, A. A.; YOUNIS M. A Framework for P2P Networking of Smart Devices Using Wi-Fi Direct. 2014 IEEE 25th Annual International Symposium on Personal, Indoor, and Mobile Radio Communication (PIMRC). Anais... . p. 2082-2087, 05-07, Set. 2014. Washington DC: IEEE. Disponível em: $<$ http://ieeexplore.ieee.org/xpl/articleDetails.jsp?arnumber=7136515>. Acesso em: 10 Out. 2015 .

9 HOANG, L. V.; OGAWA H. A platform for building ad hoc social networks based on Wi-Fi direct. 2014 IEEE 3rd Global Conference on Consumer Electronics (GCCE).

Anais... . p. 626-629, 07-10, Out. 2014. Tokyo: IEEE. Disponível em: $<$ http://ieeexplore.ieee.org/xpl/articleDetails.jsp?arnumber=7031171>. Acesso em: 10 Out. 2015.

10 HADIKS, A. et al. A Study of Stealthy Denial-of-Service Attacks in Wi-Fi Direct Device-to-Device Networks. 2014 IEEE 11th Consumer Communications and Networking 
Conference (CCNC). Anais... . p. 507-508, 10-13, Jan. 2014. Las Vegas, NV: IEEE. Disponível em: <http://ieeexplore.ieee.org/xpl/articleDetails.jsp?arnumber=6994425>. Acesso em: 10 Out. 2015.

11 WANG, Y. et al. A Wi-Fi Direct based P2P application prototype for mobile social networking in proximity (MSNP). 2014 IEEE 12th International Conference on Dependable, Autonomic and Secure Computing (DASC). Anais... . p. 283-288, 24-27, Ago. 2014. Dalian: IEEE. Disponível em:

$<$ http://ieeexplore.ieee.org/xpl/articleDetails.jsp?arnumber=6945702>. Acesso em: 10 Out. 2015.

12 TRIFUNOVIC, S. et al. Adaptive Role Switching for Fair and Efficient Battery Usage in Device-to-device Communication. ACM SIGMOBILE Mobile Computing and

Communications Review, v. 18, p. 25-36, Fev. 2014. Disponível em:

<http://dl.acm.org/citation.cfm?id=2581560>. Acesso em: 10 Out. 2015.

13 WONG, P. et al. Automatic Android-based Wireless Mesh Networks. Informatica (Slovenia), v. 38, p. 313-320, 2014. Disponível em: < https://www.scopus.com/record/display.uri?eid=2-s2.0-84920549099\&origin=resultslist $>$. Acesso em: 10 Out. 2015.

14 CULLEN, G. et al. CAPTURE - Extending the Scope of Self-Localization in Indoor Positioning Systems. 2015 International Conference on Indoor Positioning and Indoor Navigation (IPIN). Anais... . p. 1-10, 13-16, Out. 2015. Banff, AB: IEEE. Disponível em: $<$ http://ieeexplore.ieee.org/xpl/articleDetails.jsp?arnumber=7346777> . Acesso em: 20 Out. 2015.

15 CASETTI, C. et al. Content-centric Routing in Wi-Fi Direct Multi-group Networks. 2015 IEEE 16th International Symposium on a World of Wireless, Mobile and Multimedia Networks (WoWMoM). Anais... . p. 1-9, 14-17, Jun. 2015. Boston, MA: IEEE. Disponível em: <http://ieeexplore.ieee.org/xpl/articleDetails.jsp?arnumber=7158136>. Acesso em: 20 Out. 2015.

16 BISIO, I. et al. Context-awareness over transient cloud in D2D networks: energy performance analysis and evaluation. Transactions on Emerging Telecommunications Technologies, 2015. Disponível em: <https://www.scopus.com/record/display.uri?eid=2s2.0-84947447783\&origin=resultslist $>$. Acesso em: 20 Out. 2015.

17 ASHTON, N. B.; ZHANG, Q. Cool-SHARE Offload Smartphone Data by Sharing. 2014 IEEE 79th Vehicular Technology Conference (VTC Spring). Anais... . p. 1-5, 18-21, Mai. 2014. Seoul: IEEE. Disponível em:

$<$ http://ieeexplore.ieee.org/xpl/articleDetails.jsp?arnumber=7022863 >. Acesso em: 20 Out. 2015.

18 YAO, C.; ZHANG, H.; SONG, L. Demo: WiFi Multihop - Implementing Device-toDevice Local Area Networks by Android Smartphones. Proceedings of the 16th ACM International Symposium on Mobile Ad Hoc Networking and Computing. Anais... . p. 
405-406, 22-25, Jun. 2015. Hangzhou: ACM. Disponível em:

<http://dl.acm.org/citation.cfm?id=2764931>. Acesso em: 20 Out. 2015.

19 JUNG, J. Y.; KIM, D. K. Development of an Instant Meeting Android Application Using Wi-Fi Direct APIs. Communications in Computer and Information Science, v.

340, p. 107-112, 2012. Disponível em: < https://www.scopus.com/record/display.uri?eid=2s2.0-84869841397\&origin=resultslist $>$. Acesso em: 20 Out. 2015.

20 MENEGATO, U. B. et al. Dynamic Clustering in WiFi Direct Technology. Proceedings of the 12th ACM international symposium on Mobility management and wireless access.

Anais... . p. 25-29, 21-26, Set. 2014. Montreal: ACM. Disponível em:

<http://dl.acm.org/citation.cfm?id=2642682>. Acesso em: 20 Out. 2015.

21 CONTI, M. et al. Experimenting opportunistic networks with WiFi Direct. 2013 IFIP Wireless Days (WD). Anais... . p. 1-6, 13-15, Nov. 2013. Valencia: IEEE. Disponível em: $<$ http://ieeexplore.ieee.org/xpl/articleDetails.jsp?arnumber=6686501>. Acesso em: 20 Out. 2015.

22 PENG, I. et al. Implementation of Resource Integration and Application of Grouped Mobile Devices Using Wi-Fi Direct. 2015 11th International Conference on Heterogeneous Networking for Quality, Reliability, Security and Robustness (QSHINE). Anais... . p. 1-4, 19-20, Ago. 2015. Taipei: IEEE. Disponível em:

$<$ http://ieeexplore.ieee.org/xpl/articleDetails.jsp?arnumber=7332533>. Acesso em: 20 Out. 2015.

23 MARINHO, R. P.; MENEGATO U. B.; OLIVEIRA A. R. IMSN Routing on Wi-Fi Direct Enabled Devices. Proceedings of the 13th ACM International Symposium on Mobility Management and Wireless Access. Anais... . p. 31-38, 2-6, Nov. 2015. Cancun: ACM. Disponível em: <http://dl.acm.org/citation.cfm?id=2810375>. Acesso em: 20 Out. 2015 .

24 POZZA, M.; PALAZZI, C. E.; BUJARI, A. Mobile Data Offloading: An Experimental Evaluation. Proceedings of the 10th International Workshop on Mobility in the Evolving Internet Architecture. Anais... . p. 2-7, 7-11, Set. 2015. Paris: ACM. Disponível em: <http://dl.acm.org/citation.cfm?id=2795394>. Acesso em: 20 Out. 2015.

25 ZHANG, H. et al. mQual: A Mobile Peer-to-Peer Network Framework Supporting Quality of Service.2015 IEEE 35th International Conference on Distributed Computing Systems (ICDCS). Anais... . p. 754-755. 29 Jun. - 2 Jul., 2015. Columbus, OH: IEEE. Disponível em: <http://ieeexplore.ieee.org/xpl/articleDetails.jsp?arnumber=7164971>. Acesso em: 20 Out. 2015.

26 ANAND, B.; WEI, T. G. mumble - Framework for Seamless Message Transfer on Smartphones. Proceedings of the 1st International Workshop on Experiences with the Design and Implementation of Smart Objects. Anais... . p. 43-48, 7-11, Set. 2015. Paris: ACM. Disponível em: <http://dl.acm.org/citation.cfm?id=2797048>. Acesso em: 20 Out. 2015. 
27 LOMBERA, I. M. et al. Peer-to-peer publication, search and retrieval using the Android mobile platform. Computer Networks, v. 65, p. 56-72, Jun. 2014. Disponível em: <http://www.sciencedirect.com/science/article/pii/S1389128614000954>. Acesso em: 20 Out. 2015.

28 YOON, S. et al. Security Analysis of Vulnerable Wi-Fi Direct. 2012 8th International Conference on Computing and Networking Technology (ICCNT). Anais... . p. 340-343, 27-29, Ago. 2012. Gueongju: IEEE. Disponível em: <http://ieeexplore.ieee.org/xpl/articleDetails.jsp?arnumber=6418681>. Acesso em: 20 Out. 2015.

29 AMBROSIN, M. et al. Smartphone and Laptop Frameworks for Vehicular Networking Experimentation. 2013 IFIP Wireless Days (WD). Anais... . p. 1-6, 13-15, Nov. 2013. Valencia: IEEE. Disponível em:

$<$ http://ieeexplore.ieee.org/xpl/articleDetails.jsp?arnumber=6686481 >. Acesso em: 20 Out. 2015 .

30 MONARREZ, A.; SINGH, G.; BUETTNER, R. Supporting First-Responders in Infrastructure-less Environments. Procedia Engineering, v. 107, p. 34-40, 2015. Disponível em: <http://www.sciencedirect.com/science/article/pii/S1877705815010097>. Acesso em: 20 Out. 2015.

31 PENNER, T. et al. Transient Clouds Assignment and Collaborative Execution of Tasks on Mobile Devices. 2014 IEEE Global Communications Conference. Anais... . p. 28012806, 8-12, Dez. 2014. Austin, TX: IEEE. Disponível em:

<http://ieeexplore.ieee.org/xpl/articleDetails.jsp?arnumber=7037232>. Acesso em: 20 Out. 2015 .

32 MALABOCCHIA, F. et al. Using Information Centric Networking for Mobile Devices Cooperation at the Network Edge. 2015 IEEE 81st Vehicular Technology Conference (VTC Spring). Anais... . p. 1-6, 11-14, Mai. 2015. Glasgow: IEEE. Disponível em: $<$ http://ieeexplore.ieee.org/xpl/articleDetails.jsp?arnumber=7146152>. Acesso em: 20 Out. 2015 .

33 ZHANG, H.; WANG, Y.; TAN, C. C. WD2: An Improved WiFi-Direct Group Formation Protocol. Proceedings of the 9th ACM MobiCom workshop on Challenged networks. Anais... . p. 55-60, 7-11. Set. 2014. Maui: ACM. Disponível em: <http://dl.acm.org/citation.cfm?id=2645674>. Acesso em: 20 Out. 2015.

34 DUAN, Y. et al. Wi-Fi Direct Multi-group Data Dissemination for Public Safety. Proceedings of World Telecommunications Congress 2014. Anais... . p. 1-6, 1-3, Jun. 2014. Berlin: VDE. Disponível em:

$<$ http://ieeexplore.ieee.org/xpl/articleDetails.jsp?arnumber=6840013>. Acesso em: 20 Out. 2015 .

35 FENG, J.; LIU, Z.; JO, Y. Wireless Channel Loss Analysis - A Case Study Using WiFiDirect. 2014 International Wireless Communications and Mobile Computing Conference (IWCMC). Anais... . p. 244-249, 4-8, Ago. 2014. Nicosia: IEEE. Disponível em: 
$<$ http://ieeexplore.ieee.org/xpl/articleDetails.jsp?arnumber=6906364>. Acesso em: 20 Out. 2015.

36 CAMPS-MUR, D.; GARCIA-SAAVEDRA A.; SERRANO P. Device-to-device communications with Wi-Fi Direct: overview and experimentation. IEEE Wireless Communications, v. 20, p. 96-104, Jun. 2013. Disponível em:

<http://ieeexplore.ieee.org/xpl/articleDetails.jsp?arnumber=6549288>. Acesso em: $10 \mathrm{Fev}$. 2016. 